\title{
The Torelli group and congruence subgroups of the mapping class group
}

\author{
Andrew Putman*
}

July 24, 2018

\section{Introduction}

Let $\Sigma_{g, n}$ be a compact oriented genus $g$ surface with $n$ boundary components. The mapping class group of $\Sigma_{g, n}$, denoted $\operatorname{Mod}_{g, n}$, is the group of orientation-preserving diffeomorphisms of $\Sigma_{g, n}$ that restrict to the identity on $\partial \Sigma_{g, n}$, modulo isotopies that fix $\partial \Sigma_{g, n}$. The group $\operatorname{Mod}_{g, n}$ plays a fundamental role in many areas of mathematics, ranging from low-dimensional topology to algebraic geometry. At least for degrees less than about $2 g / 3$, the cohomology of $\operatorname{Mod}_{g, n}$ is well-understood due to the resolution of the Mumford conjecture by Madsen and Weiss [27] (together with Harer's unpublished improved version of his homological stability theorem; see [5] for an exposition of this).

However, the cohomology of finite-index subgroups of $\operatorname{Mod}_{g, n}$ remains a mystery. In these notes, we will focus on one low-degree calculation. Consider $n \in\{0,1\}$. For an integer $p$, the level $p$ congruence subgroup of $\operatorname{Mod}_{g, n}$, denoted $\operatorname{Mod}_{g, n}(p)$, is the subgroup of $\operatorname{Mod}_{g, n}$ consisting of mapping classes that act trivially on $\mathrm{H}_{1}\left(\Sigma_{g, n} ; \mathbb{Z} / p\right)$. Another description of $\operatorname{Mod}_{g, n}(p)$ is as follows. The action of $\operatorname{Mod}_{g, n}$ on $\mathrm{H}_{1}\left(\Sigma_{g, n} ; \mathbb{Z}\right)$ preserves the algebraic intersection pairing. Since $n \leq 1$, this is a nondegenerate alternating form, so we obtain a representation $\operatorname{Mod}_{g, n} \rightarrow \operatorname{Sp}_{2 g}(\mathbb{Z})$. Classically this representation was known to be surjective (see $\S 1.1$ ). Let $\operatorname{Sp}_{2 g}(\mathbb{Z}, p)$ be the subgroup of $\mathrm{Sp}_{2 g}(\mathbb{Z})$ consisting of matrices which equal the identity modulo $p$. Then $\operatorname{Mod}_{g, n}(p)$ is the pullback of $\mathrm{Sp}_{2 g}(\mathbb{Z}, p)$ to $\operatorname{Mod}_{g, n}$.

These notes will discuss the calculation of $\mathrm{H}^{2}\left(\operatorname{Mod}_{g, n}(p) ; \mathbb{Z}\right)$. One motivation for this is the study of line bundles on the finite cover of the moduli space of curves associated to $\operatorname{Mod}_{g, n}(p)$, which is known as the moduli space of curves with level $p$ structures. The first Chern class of such a line bundle lies in $\mathrm{H}^{2}\left(\operatorname{Mod}_{g, n}(p) ; \mathbb{Z}\right)$, and the determination of $\mathrm{H}^{2}\left(\operatorname{Mod}_{g, n}(p) ; \mathbb{Z}\right)$ is the heart of the paper [36], which gives a complete classification of such line bundles. However, in these notes we will ignore this connection to algebraic geometry. Instead, we will use the computation of this cohomology group as an excuse to discuss a number of interesting topics related to the mapping class group.

\footnotetext{
${ }^{*}$ Supported in part by NSF grant DMS-1005318
} 
The universal coefficients exact sequence for $\mathrm{H}^{2}\left(\operatorname{Mod}_{g, n}(p) ; \mathbb{Z}\right)$ takes the form

$$
\begin{aligned}
0 \longrightarrow \operatorname{Ext}\left(\mathrm{H}_{1}\left(\operatorname{Mod}_{g, n}(p) ; \mathbb{Z}\right), \mathbb{Z}\right) \longrightarrow & \mathrm{H}^{2}\left(\operatorname{Mod}_{g, n}(p) ; \mathbb{Z}\right) \\
& \longrightarrow \operatorname{Hom}\left(\mathrm{H}_{2}\left(\operatorname{Mod}_{g, n}(p) ; \mathbb{Z}\right), \mathbb{Z}\right) \longrightarrow 0
\end{aligned}
$$

The third and fourth lecture will be devoted to calculating the kernel and cokernel of this exact sequence. They will be proceeded by two lectures on necessary background. Let us now give a more detailed description of the four lectures.

- Lecture 1 will be devoted to the Torelli group. Denoted $\mathcal{I}_{g, n}$, this is the subgroup of $\operatorname{Mod}_{g, n}$ consisting of mapping classes that act trivially on $\mathrm{H}_{1}\left(\Sigma_{g, n} ; \mathbb{Z}\right)$. There are short exact sequences

$$
1 \longrightarrow \mathcal{I}_{g, n} \longrightarrow \operatorname{Mod}_{g, n} \longrightarrow \operatorname{Sp}_{2 g}(\mathbb{Z}) \longrightarrow 1
$$

and

$$
1 \longrightarrow \mathcal{I}_{g, n} \longrightarrow \operatorname{Mod}_{g, n}(p) \longrightarrow \operatorname{Sp}_{2 g}(\mathbb{Z}, p) \longrightarrow 1,
$$

and the structure of $\operatorname{Mod}_{g, n}(p)$ is a sort of mixture of the structure of $\mathcal{I}_{g, n}$ and $\mathrm{Sp}_{2 g}(\mathbb{Z}, p)$.

- Lecture 2 will be devoted to the Johnson homomorphism. Set $\mathcal{H}=\mathrm{H}_{1}\left(\Sigma_{g, n} ; \mathbb{Z}\right)$. The Johnson homomorphism is a surjective homomorphism $\tau: \mathcal{I}_{g, 1} \longrightarrow \wedge^{3} \mathcal{H}$. A deep theorem of Johnson shows that the Johnson homomorphism gives the "rational part" of the abelianization of $\mathcal{I}_{g, 1}$. More precisely, $\mathrm{H}_{1}\left(\mathcal{I}_{g, 1} ; \mathbb{Z}\right) \cong$ $W \oplus \wedge^{3} \mathcal{H}$, where $W$ consists of torsion (in fact, 2 -torsion). We will also construct a "mod $p$ " version of the Johnson homomorphism which takes the form $\tau_{p}$ : $\operatorname{Mod}_{g, 1}(p) \longrightarrow \mathcal{H}_{p}$, where $\mathcal{H}_{p}=\mathrm{H}_{1}\left(\Sigma_{g, n} ; \mathbb{Z} / p\right)$.

- Lecture 3 is devoted to calculating $\mathrm{H}_{1}\left(\operatorname{Mod}_{g, 1}(p) ; \mathbb{Z}\right)$ for odd $p$. See the beginning of that lecture for why we restrict to odd $p$ and do not consider the closed case. There are two basic pieces. The first comes from the mod $p$ Johnson homomorphism and the second comes from the abelianization of $\mathrm{Sp}_{2 g}(\mathbb{Z}, p)$.

- Lecture 4 is devoted to proving that $\mathrm{H}_{2}\left(\operatorname{Mod}_{g}(p) ; \mathbb{Q}\right) \cong \mathbb{Q}$. Of course, this implies that

$$
\operatorname{Hom}\left(\mathrm{H}_{2}\left(\operatorname{Mod}_{g, n}(p) ; \mathbb{Z}\right), \mathbb{Z}\right) \cong \mathbb{Z} .
$$

The major work here is related to homological stability.

\section{Lecture 1 : The Torelli group}

The Torelli group was first considered by Nielsen and Magnus in the early 20th century. However, its study only really took off in the late '70's and early '80's thanks to work of several people, most especially Birman and Johnson. Johnson's work has proven particularly fundamental and influential, and his survey [25] cannot be recommended enough.

Throughout this lecture, we will fix some $n \in\{0,1\}$. 

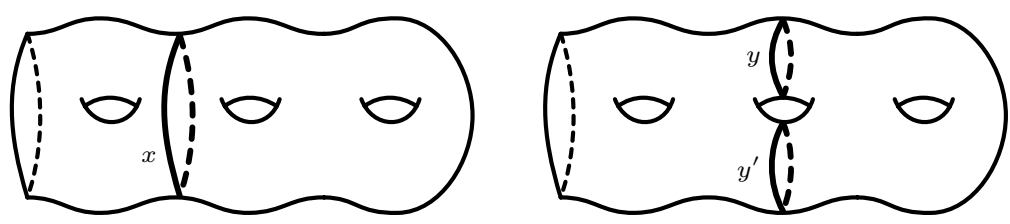

Figure 1.1: A separating twist $T_{x}$ and a bounding pair map $T_{y} T_{y^{\prime}}^{-1}$

The symplectic representation. Recall that $\mathcal{I}_{g, n}$ is the kernel of the representation $\operatorname{Mod}_{g, n} \rightarrow \operatorname{Sp}_{2 g}(\mathbb{Z})$ arising from the action of $\operatorname{Mod}_{g, n}$ on $\mathrm{H}_{1}\left(\Sigma_{g, n} ; \mathbb{Z}\right)$. We will need the following fact about this action. If $x$ is a simple closed curve on $\Sigma_{g, n}$, then let $T_{x}$ denote the right Dehn twist about $x$. Also, let $i_{a}(\cdot, \cdot)$ denote the algebraic intersection pairing on $\mathrm{H}_{1}\left(\Sigma_{g, n} ; \mathbb{Z}\right)$.

Lemma 1.1. Let $x$ be a simple closed curve on $x$. Orient $x$ in an arbitrary way, and let $[x] \in \mathrm{H}_{1}\left(\Sigma_{g, 1} ; \mathbb{Z}\right)$ denote the associated homology class. Then for $v \in \mathrm{H}_{1}\left(\Sigma_{g, 1} ; \mathbb{Z}\right)$, we have

$$
T_{x}(v)=v+i_{a}([x], v) \cdot[x] .
$$

Remark. The Dehn twist $T_{x}$ does not depend on an orientation on $x$. As a sanity check, you should verify that despite its appearance, the formula in Lemma 1.1 does not depend on the orientation of $x$.

Exercise 1.2. Prove Lemma 1.1.

Basic elements of Torelli. Lemma 1.1 allows us to construct some important elements of $\mathcal{I}_{g, 1}$. First, if $[x]=0$, then $T_{x}$ acts trivially on $\mathrm{H}_{1}\left(\Sigma_{g, 1} ; \mathbb{Z}\right)$. This will happen exactly when $x$ bounds an embedded subsurface of $x$ (see Figure 1.1). We will call such elements of Torelli separating twists. Next, the formula Lemma 1.1 only depends on the homology class of the simple closed curve. Thus if $y$ and $y^{\prime}$ are homologous, then $T_{y}$ and $T_{y^{\prime}}$ act the same on $\mathrm{H}_{1}\left(\Sigma_{g, 1} ; \mathbb{Z}\right)$, so $T_{y} T_{y^{\prime}}^{-1} \in \mathcal{I}_{g, 1}$. If $y$ and $y^{\prime}$ are disjoint and homologous, then their union bounds an embedded subsurface (see Figure 1.1). If in addition to being disjoint neither $y$ nor $y^{\prime}$ is separating, then we will call $T_{y} T_{y^{\prime}}^{-1} \in \mathcal{I}_{g, 1}$ a bounding pair map.

Generating sets for Torelli. We have the following theorem.

Theorem 1.3. For all $g \geq 1$, the group $\mathcal{I}_{g, 1}$ is generated by bounding pair maps and separating twists. For $g \geq 3$, only bounding pair maps are needed. 


\section{Remarks.}

1. The fact that $\mathcal{I}_{g, 1}$ is generated by bounding pair maps and separating twists was originally proven by Powell [31], using earlier work of Birman [3]. This proof depended on some heroic calculations in the symplectic group whose details were omitted from the published papers. More recently, Putman [32] gave a modern proof using the curve complex. Even more recently, Hatcher and Margalit [16] have given an even shorter proof.

2. The fact that for $g \geq 3$ only bounding pair maps are needed is due to Johnson [19]. He later proved a remarkable theorem which says that $\mathcal{I}_{g, 1}$ is finitely generated for $g \geq 3$ (see [22]). The size of Johnson's generating set grows exponentially in $g$. Answering a conjecture of Johnson, Putman [38] has recently constructed a generating set for $\mathcal{I}_{g, 1}$ that grows cubically in $g$. As we will discuss below, the abelianization of $\mathcal{I}_{g, 1}$ has rank cubic in the genus, so one cannot do better.

3. McCullough and Miller [28] proved that $\mathcal{I}_{2, n}$ is not finitely generated for $n \in$ $\{0,1\}$. Later, in his thesis Mess [29] proved that $\mathcal{I}_{2}$ is an infinite rank free group.

4. It is not known if $\mathcal{I}_{g, n}$ is finitely presentable for $g \geq 3$.

The Birman exact sequence. We will need to make several calculations in $\mathcal{I}_{g, n}$. For us, the key tool for making such calculations is the fact that $\mathcal{I}_{g, n}$ contains a large number of groups derived from surface groups. This follows from the Birman exact sequence, which takes the following form. Let $U \Sigma_{g}$ be the unit tangent bundle of $\Sigma_{g}$. For $g \geq 2$, we then have an exact sequence

$$
1 \longrightarrow \pi_{1}\left(U \Sigma_{g}\right) \longrightarrow \operatorname{Mod}_{g, 1} \longrightarrow \operatorname{Mod}_{g} \longrightarrow 1 .
$$

The terms here have the following meanings. Let $\beta$ be the boundary component of $\Sigma_{g, 1}$.

- The map $\operatorname{Mod}_{g, 1} \rightarrow \operatorname{Mod}_{g}$ comes from gluing a disc to $\beta$ and extending mapping classes over this disc by the identity.

- The subgroup $\pi_{1}\left(U \Sigma_{g}\right)$ of $\operatorname{Mod}_{g, 1}$ is known as the "disc-pushing subgroup". The mapping class associated to $\gamma \in \pi_{1}\left(U \Sigma_{g}\right)$ "pushes" the boundary component $\beta$ around the surface while allowing it to rotate.

Of course, the original version of the Birman exact sequence goes back to work of Birman [2]. The version here first appeared in [22]; see [12] for a textbook treatment.

The loop around the fiber. The fiber $F_{0}$ of $\mathrm{U} \Sigma_{g}$ over the basepoint satisfies $F_{0} \cong S^{1}$. The orientation on $\Sigma_{g}$ determines an orientation on $F_{0}$, so it makes sense to talk about "clockwise" and "counterclockwise" directions on $F_{0}$. The group $\pi_{1}\left(U \Sigma_{g}\right)$ contains a distinguished element $\delta_{0}$ which goes once around $F_{0}$ in the clockwise direction. The element of the disc-pushing subgroup of $\operatorname{Mod}_{g, 1}$ corresponding to $\delta_{0}$ 


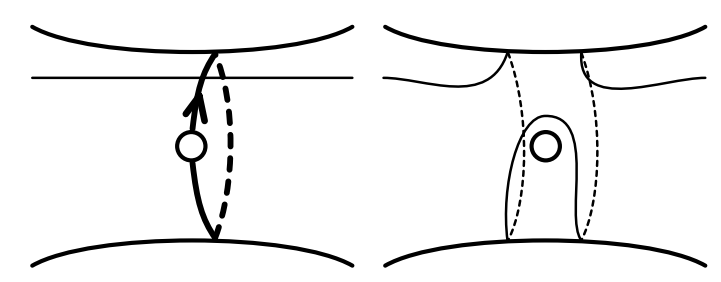

Figure 1.2: Pushing the boundary component around a simple closed curve induces a bounding pair map
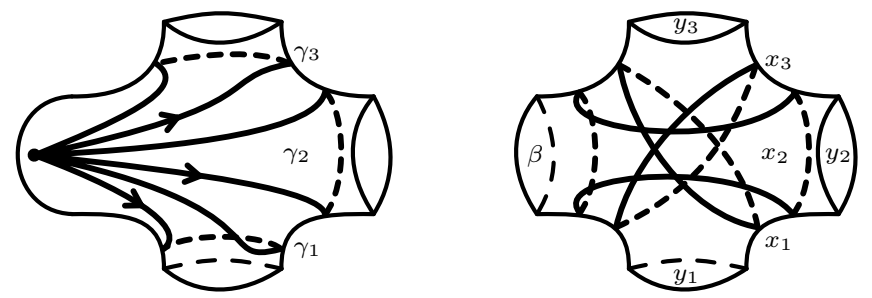

Figure 1.3: The lantern relation is $\tilde{\gamma}_{1} \cdot \tilde{\gamma}_{2} \cdot \tilde{\gamma}_{3}=\delta_{0}^{k}$. To make the figure more attractive, the curves $\gamma_{i}$ have what appears to be a singularity at the basepoint, but in reality one should imagine them rounded and smooth there. In terms of Dehn twists, the lantern relation is $\left(T_{x_{3}} T_{y_{3}}^{-1}\right)\left(T_{x_{2}} T_{y_{2}}^{-1}\right)\left(T_{x_{1}} T_{y_{1}}^{-1}\right)=T_{\beta}$

rotates the boundary component $\beta$ by a full turn in the clockwise direction. Clearly this is simply $T_{\beta}$. Observe that $T_{\beta} \in \mathcal{I}_{g, 1}$.

Calculating in the disc-pushing subgroup. Consider some $\gamma \in \pi_{1}\left(\Sigma_{g}\right)$ that can be realized by a smoothly embedded simple closed curve. The derivative of a smooth simple representative of $\gamma$ is a path in the tangent bundle of $\gamma$ which does not contain any zero vectors. For some fixed Riemannian metric on the surface, we can reparametrize $\gamma$ so that its derivative is a loop $\tilde{\gamma}$ in the unit tangent bundle. If $\gamma \neq 1$, then the element $\tilde{\gamma} \in \pi_{1}\left(U \Sigma_{g}\right)$ is independent of the choice of a smoothly embedded representative of $\gamma$. Indeed, any two such realizations are smoothly homotopic (this can be proved using the techniques in [11]; to test your understanding, you should verify that this fails if $\gamma=1$ ).

Let $\tau_{\gamma} \in \operatorname{Mod}_{g, 1}$ be the element of the disc-pushing subgroup corresponding to $\tilde{\gamma} \in \pi_{1}\left(U \Sigma_{g}\right)$. As is shown in Figure 1.2, the mapping class $\tau_{\gamma}$ is a bounding pair map, and hence lies in $\mathcal{I}_{g, 1}$. Since the loop $\delta_{0}$ around the fiber also corresponds to an element of $\mathcal{I}_{g, 1}$, we deduce that the disc-pushing subgroup lies in $\mathcal{I}_{g, 1}$. This implies that relations in $\pi_{1}\left(U \Sigma_{g}\right)$ yield relations in $\mathcal{I}_{g, 1}$. Even more relations can be obtained by embedding $\mathcal{I}_{g, 1}$ into $\mathcal{I}_{g^{\prime}, n}$ via a subsurface inclusion $\Sigma_{g, 1} \hookrightarrow \Sigma_{g^{\prime}, n}$.

As an example, consider the relation $\gamma_{1} \cdot \gamma_{2} \cdot \gamma_{3}=1$ in $\pi_{1}\left(\Sigma_{g, 1}\right)$ depicted in Figure 1.3. We have $\tilde{\gamma}_{1} \cdot \tilde{\gamma}_{2} \cdot \tilde{\gamma}_{3}=\delta_{0}^{k}$ for some $k \in \mathbb{Z}$.

Exercise 1.4. Prove that $k=1$.

The associated relation $\tau_{\gamma_{3}} \tau_{\gamma_{2}} \tau_{\gamma_{1}}=\delta_{0}$ in $\mathcal{I}_{g, 1}$ is the lantern relation

$$
\left(T_{x_{3}} T_{y_{3}}^{-1}\right)\left(T_{x_{2}} T_{y_{2}}^{-1}\right)\left(T_{x_{1}} T_{y_{1}}^{-1}\right)=T_{\beta}
$$



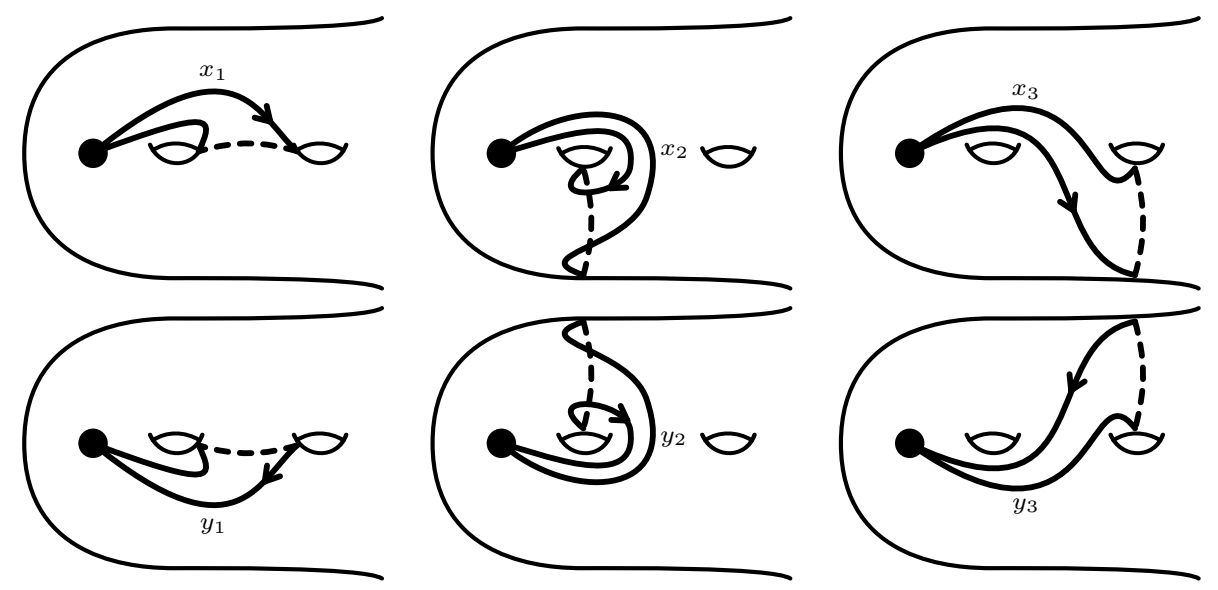

Figure 1.4: $x_{1} x_{2} x_{3}=1$ and $y_{1} y_{2} y_{3}=1$

here the curves $x_{i}$ and $y_{i}$ are as depicted in Figure 1.3.

Remark. The order of the terms in (1) is the opposite of what one might expect because elements in the fundamental group are composed left to right but mapping classes are composed right to left.

Observe that if $g^{\prime} \geq 3$, then this relation can be embedded in $\mathcal{I}_{g^{\prime}, n}$ to express a separating twist as a product of bounding pair maps (c.f. Theorem 1.3).

Killing off separating twists. The lantern relation gives numerous ways of expressing $T_{\beta}$ as a product of bounding pair maps. For $1 \leq i \leq 3$, let $x_{i}, y_{i} \in \pi_{1}\left(\Sigma_{g}\right)$ be the curves in Figure 1.4. Observe that $x_{1} x_{2} x_{3}=1$ and $y_{1} y_{2} y_{3}=1$, so we have two different lantern relations

$$
T_{\beta}=\tau_{x_{3}} \tau_{x_{2}} \tau_{x_{1}} \quad \text { and } \quad T_{\beta}=\tau_{y_{3}} \tau_{y_{2}} \tau_{y_{1}} .
$$

These curves have the property that $x_{i}$ is homologous to $y_{i}^{-1}$ for $1 \leq i \leq 3$. The group $\mathcal{I}_{g, 1}$ acts on $\pi_{1}\left(\Sigma_{g}\right)$, and it is not hard to see that there exists some $f_{i} \in \mathcal{I}_{g, 1}$ such that $f_{i}\left(x_{i}\right)=y_{i}^{-1}$. To use this, we will need the following exercise.

Exercise 1.5. If $\gamma \in \pi_{1}\left(\Sigma_{g}\right)$ can be realized by a simple closed curve and if $f \in \operatorname{Mod}_{g, 1}$, then $\tau_{f(\gamma)}=f \tau_{\gamma} f^{-1}$.

Applying Exercise 1.5 several times, we obtain the following relation in $\mathcal{I}_{g, 1}$.

$$
\begin{aligned}
T_{\beta}^{2} & =\left(\tau_{x_{3}} \tau_{x_{2}} \tau_{x_{1}}\right)\left(\tau_{y_{3}} \tau_{y_{2}} \tau_{y_{1}}\right) \\
& =\left(\tau_{x_{3}} \tau_{x_{2}} \tau_{x_{1}}\right)\left(\tau_{f_{3}\left(x_{3}\right)}^{-1} \tau_{f_{2}\left(x_{2}\right)}^{-1} \tau_{f_{1}\left(x_{1}\right)}^{-1}\right) \\
& =\left(\tau_{x_{3}} \tau_{x_{2}} \tau_{x_{1}}\right)\left(f_{3} \tau_{x_{3}}^{-1} f_{3}^{-1} f_{2} \tau_{x_{2}}^{-1} f_{2}^{-1} f_{1} \tau_{x_{1}}^{-1} f_{1}^{-1}\right)
\end{aligned}
$$

Upon abelianizing $\mathcal{I}_{g, 1}$, the right hand side of $(2)$ vanishes. Letting $\left[T_{\beta}\right] \in \mathrm{H}_{1}\left(\mathcal{I}_{g, 1} ; \mathbb{Z}\right)$ be the associated element of the abelianization, we obtain that $2\left[T_{\beta}\right]=0$.

If $x$ is a separating curve on a surface of genus at least 3 , then we can embed the above relation into the surface to get that $T_{x}^{2}$ has to vanish upon abelianizing the Torelli group. We have proven the following. 
Lemma 1.6. Fix $g \geq 3$ and $n \in\{0,1\}$. Let $T_{x}$ be a separating twist in $\mathcal{I}_{g, n}$. Then the image $\left[T_{x}\right]$ of $T_{x}$ in $\mathrm{H}_{1}\left(\mathcal{I}_{g, n} ; \mathbb{Z}\right)$ satisfies $2\left[T_{x}\right]=0$.

Lemma 1.6 first appeared in [24]. The above is a version of Johnson's proof. For an alternate exposition of that proof which arranges the details a little differently, see $[35, \S 7.2]$.

A preview. In Lecture 2, we will construct the important Johnson homomorphism. Letting $\mathcal{H}=\mathrm{H}_{1}\left(\Sigma_{g, n} ; \mathbb{Z}\right)$, this is a surjective homomorphism

$$
\tau: \mathcal{I}_{g, 1} \longrightarrow \wedge^{3} \mathcal{H} .
$$

There is also a version for closed surfaces, but we will not discuss it. The key property of the Johnson homomorphism is that its kernel is exactly the subgroup generated by separating twists. Lemma 1.6 will then allow us to deduce the following theorem of Johnson [24].

Theorem 1.7. For $g \geq 3$, we have $\mathrm{H}_{1}\left(\mathcal{I}_{g, 1} ; \mathbb{Z}\right) \cong W \oplus \wedge^{3} \mathcal{H}$, where $W$ consists of 2-torsion.

Remark. Johnson also calculated the 2-torsion $W$. The associated $\mathbb{Z} / 2$-quotients of $\mathcal{I}_{g, 1}$ come from the Rochlin invariants of homology 2 -spheres. They were originally constructed by Birman and Craggs [4]. Later, in [21] Johnson packaged all of Birman and Craggs's homomorphisms together into a single homomorphism and determined exactly how many linearly independent quotients they had constructed.

As a prologue for the construction, we recommend performing the following exercise, which explains the appearance of $\wedge^{3} \mathcal{H}$ in the Johnson homomorphism.

Exercise 1.8. Let $\mathbb{T}^{n}$ denote the $n$-torus $\left(S^{1}\right)^{n}$.

1. Prove that the cohomology ring $\mathrm{H}^{*}\left(\mathbb{T}^{n} ; \mathbb{Z}\right)$ is isomorphic to the exterior algebra $\wedge^{*} \mathbb{Z}^{n}$.

2. Let $G$ be an abelian topological group. Define a product

$$
\mathrm{H}_{i}(G ; \mathbb{Z}) \otimes \mathrm{H}_{j}(G ; \mathbb{Z}) \longrightarrow \mathrm{H}_{i+j}(G ; \mathbb{Z})
$$

via the composition

$$
\mathrm{H}_{i}(G ; \mathbb{Z}) \otimes \mathrm{H}_{j}(G ; \mathbb{Z}) \stackrel{\phi}{\longrightarrow} \mathrm{H}_{i+j}(G \times G ; \mathbb{Z}) \stackrel{\psi}{\longrightarrow} \mathrm{H}_{i+j}(G ; \mathbb{Z}),
$$

where $\phi$ is the map coming from the Künneth exact sequence and $\psi$ is induced by the group product $G \times G \rightarrow G$. Prove that with this product structure, $\mathrm{H}_{*}(G ; \mathbb{Z})$ is a graded-commutative algebra. We remark that this product is known as the Pontryagin product.

3. The space $\mathbb{T}^{n}$ is an abelian topological group. Prove that the resulting gradedcommutative ring $\mathrm{H}_{*}\left(\mathbb{T}^{n} ; \mathbb{Z}\right)$ is isomorphic to the exterior algebra $\wedge^{*} \mathbb{Z}^{n}$. 


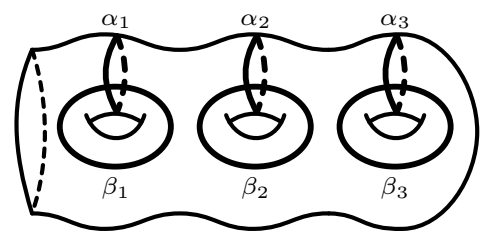

Figure 1.5: A geometric symplectic basis

\subsection{Appendix to lecture 1 : the surjectivity of the symplectic representation}

Recall that the action of $\operatorname{Mod}_{g, 1}$ on $\mathrm{H}_{1}\left(\Sigma_{g, 1} ; \mathbb{Z}\right)$ preserves the algebraic intersection pairing and thus gives a representation $\pi: \operatorname{Mod}_{g, 1} \rightarrow \operatorname{Sp}_{2 g}(\mathbb{Z})$. In this appendix, we will give a sequence of exercises about the surjectivity of $\pi$.

A symplectic basis for $\mathrm{H}_{1}\left(\Sigma_{g, 1} ; \mathbb{Z}\right)$ is a basis $\left\{a_{1}, b_{1}, \ldots, a_{g}, b_{g}\right\}$ for $\mathrm{H}_{1}\left(\Sigma_{g, 1} ; \mathbb{Z}\right)$ such that

$$
i_{a}\left(a_{i}, b_{j}\right)=\delta_{i j} \quad \text { and } \quad i_{a}\left(a_{i}, a_{j}\right)=i_{a}\left(b_{i}, b_{j}\right)=0
$$

for all $1 \leq i, j \leq g$. Let $\mathcal{S}$ be the set of symplectic bases for $\mathrm{H}_{1}\left(\Sigma_{g, 1} ; \mathbb{Z}\right)$. The following exercise should be straightforward.

Exercise 1.9. $\mathrm{Sp}_{2 g}(\mathbb{Z})$ acts simply transitively on $\mathcal{S}$.

If $x$ and $y$ are simple closed curves on $\Sigma_{g, 1}$, then let $i_{g}(x, y)$ be their geometric intersection number; i.e. the minimal cardinality of $x^{\prime} \cap y^{\prime}$ as $x^{\prime}$ and $y^{\prime}$ range over all simple closed curves homotopic to $x$ and $y$, respectively. A geometric symplectic basis (see Figure 1.5) is a collection $\left\{\alpha_{1}, \beta_{1}, \ldots, \alpha_{g}, \beta_{g}\right\}$ of simple closed curves on $\Sigma_{g, 1}$ such that

$$
i_{g}\left(\alpha_{i}, \beta_{j}\right)=\delta_{i j} \quad \text { and } \quad i_{g}\left(\alpha_{i}, \alpha_{j}\right)=i_{g}\left(\beta_{i}, \beta_{j}\right)=0
$$

for all $1 \leq i, j \leq g$. Let $\mathcal{G}$ be the set of geometric symplectic bases on $\Sigma_{g, 1}$. We then have the following.

Exercise 1.10. $\operatorname{Mod}_{g, 1}$ acts transitively on $\mathcal{G}$. Hint : given two geometric symplectic bases, prove using the Euler characteristic that you get homeomorphic surfaces when you cut along them.

The following lemma is the heart of the fact that $\pi\left(\operatorname{Mod}_{g, 1}\right)=\operatorname{Sp}_{2 g}(\mathbb{Z})$.

Lemma 1.11. If $\left\{a_{1}, b_{1}, \ldots, a_{g}, b_{g}\right\}$ is a symplectic basis for $\mathrm{H}_{1}\left(\Sigma_{g} ; \mathbb{Z}\right)$, then there exists a geometric symplectic basis $\left\{\alpha_{1}, \beta_{1}, \ldots, \alpha_{g}, \beta_{g}\right\}$ on $\Sigma_{g}$ such that $\left[\alpha_{i}\right]=a_{i}$ and $\left[\beta_{i}\right]=b_{i}$ for $1 \leq i \leq g$.

Proofs of Lemma 1.11 can be found in [32, Lemma A.3] and [12, 3rd proof of Lemma 6.4]; however, it is worthwhile to contemplate how one might prove it (though it is probably too hard for an exercise).

Exercise 1.12. Combine Lemma 1.11 with Exercises 1.9 and 1.10 to deduce that $\pi\left(\operatorname{Mod}_{g, 1}\right)=\operatorname{Sp}_{2 g}(\mathbb{Z})$. 


\section{Lecture 2 : The Johnson homomorphism}

Let $\mathcal{H}=\mathrm{H}_{1}\left(\Sigma_{g} ; \mathbb{Z}\right)$. In this lecture, we will construct the Johnson homomorphism, which is a surjective homomorphism

$$
\tau: \mathcal{I}_{g, 1} \longrightarrow \wedge^{3} \mathcal{H}
$$

This homomorphism can be constructed in a number of completely different ways. It was originally constructed in [20] by examining the action of $\mathcal{I}_{g, 1}$ on the second nilpotent truncation of $\pi_{1}\left(\Sigma_{g, 1}\right)$. We explain this original construction in an appendix. In his survey [25], Johnson outlined several alternate constructions. We will use a definition in terms of mapping tori which was introduced in [25] and was first shown to be equivalent to the original definition by Hain [13]. Our exposition will follow the paper [10] of Church and Farb, which gives a more direct proof of this equivalence.

The construction. Consider $f \in \mathcal{I}_{g, 1}$. Though it is an abuse of notation, we will regard $f$ as a homeomorphism of $\Sigma_{g, 1}$. Glue a disc to the boundary component of $\Sigma_{g, 1}$ and extend $f$ over this disc by the identity to obtain a homeomorphism $F$ of $\Sigma_{g}$. Let $p_{0} \in \Sigma_{g}$ be the center of the glued-in disc, so $F\left(p_{0}\right)=p_{0}$. Now let $M_{F}$ be the mapping torus of $F$, i.e. the quotient $\Sigma_{g} \times I / \sim$, where $(x, 1) \sim(F(x), 0)$. Give $M_{F}$ the basepoint $q_{0}=\left(p_{0}, 0\right)$.

There is a distinguished element $t \in \pi_{1}\left(M_{F}, q_{0}\right)$ which traverses the embedded loop $p_{0} \times I / \sim$ in $M_{F}$ in the positive direction. Fix a standard generating set $S=$ $\left\{s_{1}, \ldots, s_{2 g}\right\}$ for $\pi_{1}\left(\Sigma_{g}\right)$ that satisfies the surface relation

$$
\left[s_{1}, s_{2}\right] \cdots\left[s_{2 g-1}, s_{2 g}\right]=1 \text {. }
$$

Since $F\left(p_{0}\right)=p_{0}$, the map $F$ acts on $\pi_{1}\left(\Sigma_{g}, p_{0}\right)$. For $1 \leq i \leq 2 g$, let $w_{i}$ be an expression for $F_{*}\left(s_{i}\right)$ in terms of the generating set $S$. We then have a presentation

$$
\left.\pi_{1}\left(M_{F}, q_{0}\right)=\left\langle s_{1}, \ldots, s_{2 g}, t\right|\left[s_{1}, s_{2}\right] \cdots\left[s_{2 g-1}, s_{2 g}\right]=1, t s_{i} t^{-1}=w_{i} \text { for } 1 \leq i \leq 2 g\right\rangle .
$$

For $\gamma \in \pi_{1}\left(\Sigma_{g}, p_{0}\right)$, let $[\gamma] \in \mathcal{H}$ be the associated element of the abelianization. Since $F \in \mathcal{I}_{g}$, we have $\left[s_{i}\right]=\left[w_{i}\right]$ for $1 \leq i \leq 2 g$. This implies that we can define a homomorphism $\phi_{*}: \pi_{1}\left(M_{F}, q_{0}\right) \rightarrow \mathcal{H}$ such that $\phi_{*}\left(s_{i}\right)=\left[s_{i}\right]$ for $1 \leq i \leq 2 g$ and such that $\phi_{*}(t)=0$.

The space $M_{F}$ is clearly a $K\left(\pi_{1}\left(M_{F}\right), 1\right)$. Let $\mathbb{T}^{2 g}$ be the $2 g$-torus. Fix an identification of $\pi_{1}\left(\mathbb{T}^{2 g}\right)$ with $\mathcal{H}$. Since $\mathbb{T}^{2 g}$ is a $K(\mathcal{H}, 1)$, the standard properties of Eilenberg-MacLane spaces show that there is a canonical homotopy class of continuous maps $\phi: M_{F} \rightarrow \mathbb{T}^{2 g}$ inducing the homomorphism $\phi_{*}$. The space $M_{F}$ is a closed 3 -manifold, so it has a canonical class $\left[M_{F}\right] \in \mathrm{H}_{3}\left(M_{F} ; \mathbb{Z}\right)$. Define

$$
\tau(f)=\phi_{*}\left(\left[M_{F}\right]\right) \in \mathrm{H}_{3}\left(\mathbb{T}^{2 g} ; \mathbb{Z}\right) \cong \wedge^{3} \mathcal{H}
$$

The last isomorphism here comes from Exercise 1.8

Summing up, we have constructed a map $\tau: \mathcal{I}_{g, 1} \rightarrow \wedge^{3} \mathcal{H}$. The following exercise is a good test of your understanding of the above construction.

Exercise 2.1. Prove that $\tau$ is independent of all the above choices except for the identification of $\pi_{1}\left(\mathbb{T}^{2 g}\right)$ with $\mathcal{H}$ (which is fixed). Next, prove that $\tau$ is a homomorphism. 
Effect on generators. The following lemma calculates $\tau$ on the generators for $\mathcal{I}_{g, 1}$. Recall that if $S$ is a genus $h$ surface with at most 1 boundary component, then a symplectic basis for $\mathrm{H}_{1}(S ; \mathbb{Z}) \cong \mathbb{Z}^{2 h}$ is a basis $\left\{a_{1}, b_{1}, \ldots, a_{h}, b_{h}\right\}$ for $\mathrm{H}_{1}(S ; \mathbb{Z})$ such that

$$
i_{a}\left(a_{i}, b_{j}\right)=\delta_{i j} \quad \text { and } \quad i_{a}\left(a_{i}, a_{j}\right)=i_{a}\left(b_{i}, b_{j}\right)=0
$$

for all $1 \leq i, j \leq h$. Here $i_{a}(\cdot, \cdot)$ is the algebraic intersection form.

\section{Lemma 2.2.}

1. Let $T_{x} \in \mathcal{I}_{g, 1}$ be a separating twist. Then $\tau\left(T_{x}\right)=0$.

2. Let $T_{x} T_{x^{\prime}}^{-1}$ be a bounding pair map on $\mathcal{I}_{g, 1}$. Let $S$ be the component of $\Sigma_{g, 1}$ cut along $x \cup x^{\prime}$ that does not contain $\partial \Sigma_{g, 1}$, so $S \cong \Sigma_{h, 2}$ for some $h<g$. Let $S^{\prime} \subset S$ be an embedded subsurface such that $S^{\prime} \cong \Sigma_{h, 1}$ and let $\left\{a_{1}, b_{1}, \ldots, a_{h}, b_{h}\right\}$ be a symplectic basis for $\mathrm{H}_{1}\left(S^{\prime} ; \mathbb{Z}\right) \subset \mathrm{H}_{1}\left(\Sigma_{g, 1} ; \mathbb{Z}\right)$. Then

$$
\tau\left(T_{x} T_{x^{\prime}}^{-1}\right)= \pm[x] \wedge\left(a_{1} \wedge b_{1}+\cdots+a_{h} \wedge b_{g}\right) .
$$

We will discuss the proof of this lemma at the end of this section. Right now, we suggest doing the following two exercises.

Exercise 2.3. Prove that the formula in Lemma 2.2 is independent of the choice of $S^{\prime}$ and its symplectic basis.

Exercise 2.4. Using Lemma 2.2, prove that $\tau$ is surjective.

Johnson's theorem. In [23], Johnson proved the following deep theorem, which is a sort of converse to part 1 of Lemma 2.2. For an alternate proof, see [35].

Theorem 2.5. The kernel of $\tau$ is generated by separating twists.

As we indicated at the end of Lecture 1, this theorem together with Lemma 1.6 implies that $\mathrm{H}_{1}\left(\mathcal{I}_{g, 1} ; \mathbb{Z}\right) \cong W \oplus \wedge^{3} \mathcal{H}$ for $g \geq 3$, where $W$ consists of 2 -torsion.

The Johnson homomorphism $\bmod p$. Set $\mathcal{H}_{p}=\mathrm{H}_{1}\left(\Sigma_{g, 1} ; \mathbb{Z} / p\right)$. We wish to construct a "mod $p$ " Johnson homomorphism

$$
\tau_{p}: \operatorname{Mod}_{g, n}(p) \rightarrow \mathcal{H}_{p}
$$

The construction goes exactly like the construction of the ordinary Johnson homomorphism. Consider $f \in \operatorname{Mod}_{g, 1}(p)$. Regard $f$ as a homeomorphism of $\Sigma_{g, 1}$. Glue a disc to the boundary component of $\Sigma_{g, 1}$ and extend $f$ over this disc by the identity to obtain a homeomorphism $F$ of $\Sigma_{g}$. Let $p_{0} \in \Sigma_{g}$ be the center of the glued-in disc, so $F\left(p_{0}\right)=p_{0}$. Let $M_{F}$ be the mapping torus of $F$ and let $q_{0}=\left(p_{0}, 0\right)$ be the basepoint for $M_{F}$.

There is a distinguished element $t \in \pi_{1}\left(M_{F}, q_{0}\right)$ which traverses the embedded loop $p_{0} \times I / \sim$ in $M_{F}$ in the positive direction. Fix a standard generating set $S=$ $\left\{s_{1}, \ldots, s_{2 g}\right\}$ for $\pi_{1}\left(\Sigma_{g}\right)$ that satisfies the surface relation

$$
\left[s_{1}, s_{2}\right] \cdots\left[s_{2 g-1}, s_{2 g}\right]=1 \text {. }
$$


Since $F\left(p_{0}\right)=p_{0}$, the map $F$ acts on $\pi_{1}\left(\Sigma_{g}, p_{0}\right)$. For $1 \leq i \leq 2 g$, let $w_{i}$ be an expression for $F_{*}\left(s_{i}\right)$ in terms of the generating set $S$. We then have a presentation

$$
\left.\pi_{1}\left(M_{F}, q_{0}\right)=\left\langle s_{1}, \ldots, s_{2 g}, t\right|\left[s_{1}, s_{2}\right] \cdots\left[s_{2 g-1}, s_{2 g}\right]=1, t s_{i} t^{-1}=w_{i} \text { for } 1 \leq i \leq 2 g\right\rangle .
$$

For $\gamma \in \pi_{1}\left(\Sigma_{g}, p_{0}\right)$, let $[\gamma]_{p} \in \mathcal{H}_{p}$ be the associated element. Since $F \in \operatorname{Mod}_{g}(p)$, we have $\left[s_{i}\right]_{p}=\left[w_{i}\right]_{p}$ for $1 \leq i \leq 2 g$. This implies that we can define a homomorphism $\phi_{*}: \pi_{1}\left(M_{F}, q_{0}\right) \rightarrow \mathcal{H}_{p}$ such that $\phi_{*}\left(s_{i}\right)=\left[s_{i}\right]_{p}$ for $1 \leq i \leq 2 g$ and such that $\phi_{*}(t)=0$.

The space $M_{F}$ is clearly a $K\left(\pi_{1}\left(M_{F}\right), 1\right)$. Let $Z$ be a $K\left(\mathcal{H}_{p}, 1\right)$. The standard properties of Eilenberg-MacLane spaces show that there is a canonical homotopy class of continuous maps $\phi: M_{F} \rightarrow Z$ inducing the homomorphism $\phi_{*}$. Define

$$
\tau_{p}^{\prime}(f)=\phi_{*}\left(\left[M_{F}\right]\right) \in \mathrm{H}_{3}(Z ; \mathbb{Z}) \cong \mathrm{H}_{3}\left(\mathcal{H}_{p} ; \mathbb{Z}\right),
$$

where $M_{F} \in \mathrm{H}_{3}\left(M_{F} ; \mathbb{Z}\right)$ is the canonical class. It is not true that $\mathrm{H}_{3}\left(\mathcal{H}_{p} ; \mathbb{Z}\right) \cong$ $\wedge^{3} \mathcal{H}_{p}$; however, $\mathrm{H}_{3}\left(\mathcal{H}_{p} ; \mathbb{Z}\right)$ does contain $\wedge^{3} \mathcal{H}_{p}$ as a direct factor (see, for example, [8, Theorem V.6.4]). Let $\tau_{p}: \operatorname{Mod}_{g, 1}(p) \rightarrow \wedge^{3} \mathcal{H}_{p}$ be the composition of $\tau_{p}^{\prime}$ with some (fixed for all time) projection of $\mathrm{H}_{3}\left(\mathcal{H}_{p} ; \mathbb{Z}\right)$ onto $\wedge^{3} \mathcal{H}_{p}$. By the same argument used in Exercise 2.1, the map $\tau_{p}$ is a well-defined homomorphism.

From its construction, it is clear that the following diagram commutes.

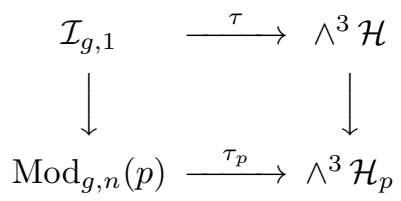

Since $\tau$ is surjective for $g \geq 3$, we obtain the following.

Theorem 2.6. The map $\tau_{p}: \operatorname{Mod}_{g, 1}(p) \rightarrow \mathcal{H}_{p}$ is surjective for $g \geq 3$.

Images of mapping tori. To prove Lemma 2.2, one would need to be able to calculate the images of fundamental classes of mapping tori under maps to a torus. Instead of giving the details of the proof, we will prove an easier theorem which illustrates how one can do this. For the proof of Lemma 2.2, see [10].

The result we will prove is as follows. In its statement and proof, we will use the natural identification of the graded-commutative algebra structure on $\mathrm{H}_{*}\left(\mathbb{T}^{n} ; \mathbb{Z}\right)$ with $\wedge^{*} \mathbb{Z}^{n}$ which was identified in Exercise 1.8.

Theorem 2.7. Let $\phi: \Sigma_{g} \rightarrow \mathbb{T}^{n}$ be a continuous map. Choose a standard basis $\left\{\alpha_{1}, \beta_{1}, \ldots, \alpha_{g}, \beta_{g}\right\}$ for $\pi_{1}\left(\Sigma_{g}\right)$, so

$$
\pi_{1}\left(\Sigma_{g}\right)=\left\langle\alpha_{1}, \beta_{1}, \ldots, \alpha_{g}, \beta_{g} \mid\left[\alpha_{1}, \beta_{1}\right] \cdots\left[\alpha_{g}, \beta_{g}\right]=1\right\rangle .
$$

Then

$$
\phi_{*}\left(\left[\Sigma_{g}\right]\right)= \pm \sum_{i=1}^{g} \phi_{*}\left(\alpha_{i}\right) \wedge \phi_{*}\left(\beta_{i}\right) \in \wedge^{2} \mathbb{Z}^{n} \cong \mathrm{H}_{2}\left(\mathbb{T}^{n} ; \mathbb{Z}\right)
$$




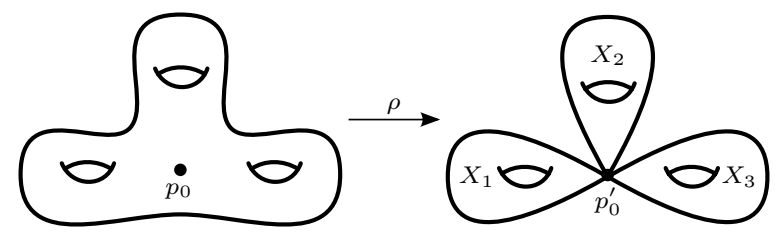

Figure 2.1: Degenerating our surface to a wedge of tori

Proof. Assume first that $g=1$, so $\Sigma_{g} \cong \mathbb{T}^{2}$. We will identify $\Sigma_{g}$ with $\mathbb{T}^{2}$. Observe that $\pi_{1}\left(\mathbb{T}^{2}\right) \cong \mathbb{Z}^{2}$ and $\mathrm{H}_{*}\left(\mathbb{T}^{2} ; \mathbb{Z}\right) \cong \wedge^{*}\left(\mathbb{Z}^{2}\right)$. Moreover, $\mathrm{H}_{2}\left(\mathbb{T}^{2} ; \mathbb{Z}\right) \cong \wedge^{2} \mathbb{Z}^{2}$ is generated by $\alpha_{1} \wedge \beta_{1}$, so $\left[\mathbb{T}^{2}\right]= \pm \alpha_{1} \wedge \beta_{1}$. By the naturality of the Pontryagin product we have

$$
\phi_{*}\left(\left[\mathbb{T}^{2}\right]\right)= \pm \phi_{*}\left(\alpha_{1}\right) \wedge \phi_{*}\left(\beta_{1}\right) .
$$

Now assume that $g>1$. We will reduce to the case $g=1$ by a method that will be familiar to algebraic geometers. Namely, we will "degenerate" our surface to a nodal surface and make the computation there. Fix a basepoint $p_{0}$ for $\Sigma_{g}$. As shown in Figure 2.1, let $\left(X, p_{0}^{\prime}\right)$ be the result of collapsing $\left(\Sigma_{g}, p_{0}\right)$ to the wedge of $g$ tori $X_{1}, \ldots, X_{g}$. Choose $X$ such that under the collapse map $\rho: \Sigma_{g} \rightarrow X$, the curves $\alpha_{i}$ and $\beta_{i}$ map to generators for $\pi_{1}\left(X_{i}, p_{0}^{\prime}\right) \subset \pi_{1}\left(X, p_{0}^{\prime}\right)$.

Exercise 2.8. Prove that the map $\phi_{*}: \pi_{1}\left(\Sigma_{g}, p_{0}\right) \rightarrow \pi_{1}\left(\mathbb{T}^{n}\right)$ factors as

$$
\pi_{1}\left(\Sigma_{g}, p_{0}\right) \stackrel{\rho_{*}}{\longrightarrow} \pi_{1}\left(X, p_{0}^{\prime}\right) \stackrel{\phi_{*}^{\prime}}{\longrightarrow} \pi_{1}\left(\mathbb{T}^{n}\right)
$$

for some homomorphism $\phi_{*}^{\prime}: \pi_{1}\left(X, p_{0}^{\prime}\right) \rightarrow \pi_{1}\left(\mathbb{T}^{n}\right)$. Hint $: \pi_{1}\left(\mathbb{T}^{n}\right)$ is abelian.

Since the spaces $\Sigma_{g}$ and $X$ and $\mathbb{T}^{n}$ are all Eilenberg-MacLane spaces, there exists some continuous map $\phi^{\prime}: X \rightarrow \mathbb{T}^{n}$ inducing $\phi_{*}^{\prime}$ such that $\phi$ is homotopic to $\phi^{\prime} \circ \rho$.

Observe that $\rho_{*}\left(\left[\Sigma_{g}\right]\right)=\left[X_{1}\right]+\cdots+\left[X_{g}\right]$. Moreover, $\rho_{*}\left(\alpha_{i}\right)$ and $\rho_{*}\left(\beta_{i}\right)$ are a standard basis for $\pi_{1}\left(X_{i}\right) \subset \pi_{1}(X)$. By the case $g=1$, we have

$$
\left(\left.\phi^{\prime}\right|_{X_{i}}\right)_{*}\left(\left[X_{i}\right]\right)= \pm \phi_{*}^{\prime}\left(\rho_{*}\left(\alpha_{i}\right)\right) \wedge \phi_{*}^{\prime}\left(\rho_{*}\left(\beta_{i}\right)= \pm \phi_{*}\left(\alpha_{i}\right) \wedge \phi_{*}\left(\beta_{i}\right) .\right.
$$

All the \pm signs are identical. Adding everything up, we get the desired result.

\section{Appendix to lecture 2 : the original construction of the Johnson homomorphism}

The original construction of the Johnson homomorphism gave a homomorphism

$$
\tau: \mathcal{I}_{g, 1} \rightarrow \operatorname{Hom}\left(\mathcal{H}, \wedge^{2} \mathcal{H}\right) .
$$

Johnson then calculated $\tau$ on generators and showed that its image was isomorphic to $\wedge^{3} \mathcal{H}$. In this appendix, we will guide you through Johnson's original construction.

If $G$ is a group, then let $\gamma_{k}(G)$ be the $k^{\text {th }}$ term in its lower central series, which is the inductively defined sequence of groups

$$
\gamma_{0}(G)=G \quad \text { and } \quad \gamma_{k+1}(G)=\left[\gamma_{k}(G), G\right] .
$$


Set $\pi=\pi_{1}\left(\Sigma_{g, 1}, *\right)$, where $* \in \partial \Sigma_{g, 1}$. We will need the following fact. We will state it as an exercise, but you should only attempt it if you know some of the basics of group cohomology (otherwise, treat it as a black box).

Exercise 2.9. Prove that $\gamma_{1}(\pi) / \gamma_{2}(\pi) \cong \wedge^{2} \mathcal{H}$. Hint : Apply the 5 -term exact sequence in group homology to the short exact sequence

$$
1 \longrightarrow \gamma_{1}(\pi) \longrightarrow \pi \longrightarrow \mathcal{H} \longrightarrow 1 \text {. }
$$

You will need the fact that $\mathrm{H}_{2}(\mathcal{H} ; \mathbb{Z}) \cong \wedge^{2} \mathcal{H}$.

Now consider the short exact sequence

$$
1 \longrightarrow \gamma_{1}(\pi) \longrightarrow \pi \longrightarrow \mathcal{H} \longrightarrow 1 \text {. }
$$

To simplify this, we mod out by $\gamma_{2}(\pi)<\gamma_{1}(\pi)$. By the above exercise, we get a short exact sequence

$$
1 \longrightarrow \wedge^{2} \mathcal{H} \longrightarrow \Gamma \longrightarrow \mathcal{H} \longrightarrow 1,
$$

where $\Gamma=\pi / \gamma_{2}(\pi)$.

Exercise 2.10. The subgroup $\mathcal{H}<\Gamma$ is central.

The mapping class group acts on $\pi$ (this is where we use the fact that the basepoint is on the boundary component, so it is fixed by the mapping class group). This action preserves $\gamma_{k}(\pi)$ for all $\pi$ (indeed, all automorphisms of $\pi$ do this!). We thus get an action of $\operatorname{Mod}_{g, 1}$ on $\Gamma$ which preserves $\wedge^{2} \mathcal{H}<\Gamma$.

Restrict this action to $\mathcal{I}_{g, 1}$. The group $\mathcal{I}_{g, 1}$ acts trivially on $\mathcal{H}$.

Exercise 2.11. The action of $\mathcal{I}_{g, 1}$ on $\wedge^{2} \mathcal{H}<\Gamma$ is trivial. This looks obvious, but you have to trace through the above definitions to see that the action of $\operatorname{Mod}_{g, 1}$ on $\wedge^{2} \mathcal{H}<\Gamma$ is what you think it is.

Fix $f \in \mathcal{I}_{g, 1}$. For $x \in \Gamma$, observe that $f(x)$ and $x$ project to the same element of $\mathcal{H}$, so $x(f(x))^{-1} \in \wedge^{2} \mathcal{H}$. Define a set map $J_{f}^{\prime}: \Gamma \rightarrow \wedge^{2} \mathcal{H}$ by $J_{f}^{\prime}(x)=x(f(x))^{-1}$.

Exercise 2.12. The map $J_{f}^{\prime}$ factors through a set map $J_{f}: \mathcal{H} \rightarrow \wedge^{2} \mathcal{H}$.

Exercise 2.13. The set map $J_{f}$ is a homomorphism.

We can thus define a set map

$$
\tau: \mathcal{I}_{g, 1} \rightarrow \operatorname{Hom}\left(\mathcal{H}, \wedge^{2} \mathcal{H}\right)
$$

by $\tau(f)=J_{f}$.

Exercise 2.14. $\tau$ is a homomorphism.

\section{Lecture 3 : The abelianization of $\operatorname{Mod}_{g, n}(p)$}

In this lecture, we calculate $\mathrm{H}_{1}\left(\operatorname{Mod}_{g, n}(p) ; \mathbb{Z}\right)$. To simplify our exposition, we will do the following.

- We will only consider the case of $\operatorname{Mod}_{g, 1}(p)$. The case of $\operatorname{Mod}_{g}(p)$ can be dealt with in a similar way, but there are a few added complications. 
- We will only consider the case where $p$ is odd. This greatly simplifies both the statements of the results and their proofs.

Main theorem. Our main theorem is as follows.

Theorem 3.1. Fix $g, p \geq 3$ such that $p$ is odd. Set $\mathcal{H}_{p}=\mathrm{H}_{1}\left(\Sigma_{g, 1} ; \mathbb{Z} / p\right)$. There is then a short exact sequence

$$
0 \longrightarrow \wedge^{3} \mathcal{H}_{p} \longrightarrow \mathrm{H}_{1}\left(\operatorname{Mod}_{g, 1}(p) ; \mathbb{Z}\right) \longrightarrow \mathrm{H}_{1}\left(\mathrm{Sp}_{2 g}(\mathbb{Z}, p) ; \mathbb{Z}\right) \longrightarrow 0 .
$$

Remarks.

1. Theorem 3.1 was proven independently by Putman [33] and Sato [39]. At the same time, Perron [30] calculated $\mathrm{H}_{1}\left(\operatorname{Mod}_{g, 1}(p) ; \mathbb{Z}\right)$ up to a 2-torsion ambiguity.

2. Sato [39] also calculated $\mathrm{H}_{1}\left(\operatorname{Mod}_{g, 1}(2) ; \mathbb{Z}\right)$. The answer is more complicated than that given in Theorem 3.1. This was later extended by Putman [36] to $\mathrm{H}_{1}\left(\operatorname{Mod}_{g, 1}(p) ; \mathbb{Z}\right)$ for $p$ not divisible by 4 . The case where $p$ is divisible by 4 is still open - see the introduction of [36] for a discussion.

3. It was originally proven by Hain [13] that $\mathrm{H}_{1}\left(\operatorname{Mod}_{g, 1}(p)\right.$; $\left.\mathbb{Z}\right)$ is finite for $g \geq 3$.

4. At the end of this lecture, we will calculate $\mathrm{H}_{1}\left(\mathrm{Sp}_{2 g}(\mathbb{Z}, p) ; \mathbb{Z}\right)$.

Beginning of the proof. Of course, Theorem 3.1 is derived from the short exact sequence

$$
1 \longrightarrow \mathcal{I}_{g, 1} \longrightarrow \operatorname{Mod}_{g, 1}(p) \longrightarrow \operatorname{Sp}_{2 g}(\mathbb{Z}, p) \longrightarrow 1 .
$$

We will need the following exercise.

Exercise 3.2. Consider a short exact sequence

$$
1 \longrightarrow G_{1} \longrightarrow G_{2} \longrightarrow G_{3} \longrightarrow 1
$$

of groups. There is then a short exact sequence

$$
0 \longrightarrow V \longrightarrow \mathrm{H}_{1}\left(G_{2} ; \mathbb{Z}\right) \longrightarrow \mathrm{H}_{1}\left(G_{3} ; \mathbb{Z}\right) \longrightarrow 0
$$

where $V$ is the image of $\mathrm{H}_{1}\left(G_{1} ; \mathbb{Z}\right)$ in $\mathrm{H}_{1}\left(G_{2} ; \mathbb{Z}\right)$.

Applying Exercise 3.2, we see that it is enough to prove that the image of $\mathrm{H}_{1}\left(\mathcal{I}_{g, 1} ; \mathbb{Z}\right)$ in $\mathrm{H}_{1}\left(\operatorname{Mod}_{g, 1}(p) ; \mathbb{Z}\right)$ is $\wedge^{3} \mathcal{H}_{p}$. The key to this is the following lemma.

Lemma 3.3. For $g \geq 3$, let $v \in \mathrm{H}_{1}\left(\operatorname{Mod}_{g, 1}(p) ; \mathbb{Z}\right)$ be in the image of $\mathrm{H}_{1}\left(\mathcal{I}_{g, 1} ; \mathbb{Z}\right)$. Then $p \cdot v=0$.

Before proving Lemma 3.3, let use it to finish the proof of Theorem 3.1. Theorem 1.7 says that

$$
\mathrm{H}_{1}\left(\mathcal{I}_{g, 1} ; \mathbb{Z}\right) \cong W \oplus \wedge^{3} \mathcal{H},
$$



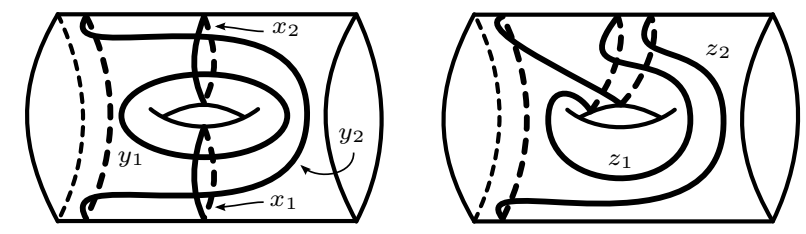

Figure 3.1: The crossed lantern relation $\left(T_{y_{1}} T_{y_{2}}^{-1}\right)\left(T_{x_{1}} T_{x_{2}}^{-1}\right)=T_{z_{1}} T_{z_{2}}^{-1}$.

where $W$ consists of 2 -torsion. Lemma 3.3 and the fact that $p$ is odd imply that the inclusion map $\mathrm{H}_{1}\left(\mathcal{I}_{g, 1} ; \mathbb{Z}\right) \rightarrow \mathrm{H}_{1}\left(\operatorname{Mod}_{g, 1}(p) ; \mathbb{Z}\right)$ factors through

$$
(W / p \cdot W) \oplus\left(\wedge^{3} \mathcal{H}\right) /\left(p \cdot \wedge^{3} \mathcal{H}\right)=\wedge^{3} \mathcal{H}_{p} .
$$

Theorem 2.6 says that the image of $\mathrm{H}_{1}\left(\mathcal{I}_{g, 1} ; \mathbb{Z}\right)$ in $\mathrm{H}_{1}\left(\operatorname{Mod}_{g, 1}(p) ; \mathbb{Z}\right)$ contains $\wedge^{3} \mathcal{H}_{p}$, so we conclude that the image equals $\mathcal{H}_{p}$, as desired.

The crossed lantern relation. To prove Lemma 3.3, we will need the following relation the mapping class group, which is known as the crossed lantern relation.

Lemma 3.4. Let $x_{i}$ and $y_{i}$ and $z_{i}$ be the curves in Figure 3.1. Then

$$
\left(T_{y_{1}} T_{y_{2}}^{-1}\right)\left(T_{x_{1}} T_{x_{2}}^{-1}\right)=T_{z_{1}} T_{z_{2}}^{-1} .
$$

Proof. This rests on two key facts:

$$
T_{x_{2}}\left(y_{i}\right)=z_{i} \quad \text { and } \quad T_{y_{2}} T_{y_{1}}^{-1}\left(x_{2}\right)=x_{1} .
$$

Repeatedly using the fact that $f T_{c} f^{-1}=T_{f(c)}$ for any simple closed curve $c$ and any mapping class $f$ (which can be proven exactly like Exercise 1.5), we calculate

$$
\begin{aligned}
T_{x_{1}} T_{x_{2}}^{-1} & =\left(\left(T_{y_{2}} T_{y_{1}}^{-1}\right) T_{x_{2}}\left(T_{y_{1}} T_{y_{2}}^{-1}\right)\right) T_{x_{2}}^{-1} \\
& \left.=\left(T_{y_{2}} T_{y_{1}}^{-1}\right)\left(T_{x_{2}}\left(T_{y_{1}} T_{y_{2}}^{-1}\right) T_{x_{2}}^{-1}\right)\right) \\
& =T_{y_{2}} T_{y_{1}}^{-1} T_{z_{1}} T_{z_{2}}^{-1} .
\end{aligned}
$$

Rearranging this gives the desired relation.

Exercise 3.5. Give an alternate derivation of the crossed lantern relation by the same technique we used to construct the lantern relation in Lecture 1. Hint : The lantern relation comes from a relation $x y z=1$ between simple closed curve in a surface group. Try to write down a different relation of the form $x y=z$ between simple closed curves. If you get stuck, see [34, §3.1.3].

The proof of Lemma 3.3. We are now in a position to prove Lemma 3.3. For $f \in \operatorname{Mod}_{g, 1}(p)$, let $[f]$ be the corresponding element of $\mathrm{H}_{1}\left(\operatorname{Mod}_{g, 1}(p) ; \mathbb{Z}\right)$. Theorem 1.3 says that $\mathcal{I}_{g, 1}$ is generated by bounding pair maps. Letting $T_{x_{1}} T_{x_{2}}^{-1}$ be a bounding pair map, it is thus enough to show that $p \cdot\left[T_{x_{1}} T_{x_{2}}^{-1}\right]=0$. There exists an embedded subsurface $S$ of $\Sigma_{g, 1}$ containing $\left\{x_{1}, x_{2}\right\}$ such that $S \cong \Sigma_{1,2}$ and such that the curves 
$\left\{x_{1}, x_{2}\right\}$ are embedded in $S$ as depicted in Figure 3.1. Let $\left\{y_{1}, y_{2}\right\}$ and $\left\{z_{1}, z_{2}\right\}$ be the curves in $S$ depicted in Figure 3.1. We thus have a crossed lantern relation

$$
\left(T_{y_{1}} T_{y_{2}}^{-1}\right)\left(T_{x_{1}} T_{x_{2}}^{-1}\right)=T_{z_{1}} T_{z_{2}}^{-1}
$$

Also, $z_{i}=T_{x_{2}}\left(y_{i}\right)$ for $i=1,2$.

The key observation is that for all $k \geq 0$, conjugating (4) by $T_{x_{2}}^{k}$ results in another crossed lantern relation

$$
\left(T_{T_{x_{2}}^{k}\left(y_{1}\right)} T_{T_{x_{2}}^{k}\left(y_{2}\right)}^{-1}\right)\left(T_{x_{1}} T_{x_{2}}^{-1}\right)=\left(T_{T_{x_{2}}^{k+1}\left(y_{1}\right)} T_{T_{x_{2}}^{n+1}\left(y_{2}\right)}^{-1}\right) .
$$

Since $T_{x_{2}}^{p} \in \operatorname{Mod}_{g, 1}(p)$, we conclude that $\left[T_{y_{1}} T_{y_{2}}^{-1}\right]$ is equal in $\mathrm{H}_{1}\left(\operatorname{Mod}_{g, 1}(p) ; \mathbb{Z}\right)$ to

$$
\begin{aligned}
{\left[T_{x_{2}}^{p}\left(T_{y_{1}} T_{y_{2}}^{-1}\right) T_{x_{2}}^{-p}\right] } & =\left[\left(T_{T_{x_{2}}^{p}\left(y_{1}\right)} T_{T_{x_{2}}^{p}\left(y_{2}\right)}^{-1}\right)\right] \\
& =\left[T_{x_{1}} T_{x_{2}}^{-1}\right]+\left[\left(T_{T_{x_{2}}^{p-1}\left(y_{1}\right)} T_{T_{x_{2}}^{p-1}\left(y_{2}\right)}^{-1}\right)\right] \\
& =2\left[T_{x_{1}} T_{x_{2}}^{-1}\right]+\left[\left(T_{T_{x_{2}}^{p-2}\left(y_{1}\right)} T_{T_{x_{2}}^{p-2}\left(y_{2}\right)}^{-1}\right)\right] \\
& \vdots \\
& =p \cdot\left[T_{x_{1}} T_{x_{2}}^{-1}\right]+\left[T_{y_{1}} T_{y_{2}}^{-1}\right],
\end{aligned}
$$

so $p \cdot\left[T_{x_{1}} T_{x_{2}}^{-1}\right]=0$, as desired.

The abelianization of $\mathrm{Sp}_{2 g}(\mathbb{Z}, p)$. To complete our description of the abelianization of $\operatorname{Mod}_{g, 1}(p)$, we need a description of $\mathrm{H}_{1}\left(\mathrm{Sp}_{2 g}(\mathbb{Z}, p) ; \mathbb{Z}\right)$. We first need some notation. Denote the $n \times n$ identity matrix by $\mathbb{I}_{n}$ and the $n \times n$ zero matrix by $\mathbb{O}_{n}$. Let $\Omega_{g}$ be the $2 g \times 2 g$ matrix $\left(\begin{array}{cc}\mathbb{O}_{g} & \mathbb{I}_{g} \\ -\mathbb{I}_{g} & \mathbb{O}_{g}\end{array}\right)$. By definition, for a commutative ring $R$ the group $\mathrm{Sp}_{2 g}(R)$ consists of all $2 g \times 2 g$ matrices $M$ with entries in $R$ such that $M^{t} \Omega_{g} M=\Omega_{g}$. Define $\mathfrak{s p}_{2 g}(R)$ to be the additive group of $2 g \times 2 g$ matrices $A$ with entries in $R$ such that $A^{t} \Omega_{g}+\Omega_{g} A=0$.

We then have the following theorem.

Theorem 3.6. Fix $g, p \geq 3$ such that $p$ is odd. Then

$$
\mathrm{H}_{1}\left(\mathrm{Sp}_{2 g}(\mathbb{Z}, p) ; \mathbb{Z}\right) \cong \mathfrak{s p}_{2 g}(\mathbb{Z} / p) \quad \text { and } \quad\left[\operatorname{Sp}_{2 g}(\mathbb{Z}, p), \operatorname{Sp}_{2 g}(\mathbb{Z}, p)\right]=\operatorname{Sp}_{2 g}\left(\mathbb{Z}, p^{2}\right) .
$$

Remarks.

1. Theorem 3.6 was proved independently by Perron [30], Putman [33], and Sato [39]. All three papers were inspired by a basic result of Lee and Szczarba [26] which calculates the abelianizations of congruence subgroups of $\mathrm{SL}_{n}(\mathbb{Z})$. See Exercise 3.9 for a discussion of Lee and Szczarba's work.

2. For $p$ even, Sato [39] proved that there is a short exact sequence

$$
0 \longrightarrow \mathcal{H}_{2} \longrightarrow \mathrm{H}_{1}\left(\mathrm{Sp}_{2 g}(\mathbb{Z}, p) ; \mathbb{Z}\right) \longrightarrow \mathfrak{s p}_{2 g}(\mathbb{Z} / p) \longrightarrow 0 .
$$

Here $\mathcal{H}_{2}=\mathrm{H}_{1}\left(\Sigma_{g, 1} ; \mathbb{Z} / 2\right)$. 
The map from $\mathrm{Sp}_{2 g}(\mathbb{Z}, p)$ to $\mathfrak{s p}_{2 g}(\mathbb{Z} / p)$. To prove Theorem 3.6, we first construct a surjective homomorphism

$$
\psi: \mathrm{Sp}_{2 g}(\mathbb{Z}, p) \longrightarrow \mathfrak{s p}_{2 g}(\mathbb{Z} / p) .
$$

Consider $M \in \mathrm{Sp}_{2 g}(\mathbb{Z}, p)$. By definition, we can write $M=\mathbb{I}_{2 g}+p \cdot A$ for some matrix $A$. Define $\psi(M)=A$ modulo $p$. We have $\psi(M) \in \mathfrak{s p}_{2 g}(\mathbb{Z} / p)$; indeed, by definition we have $\Omega_{g}=M^{t} \Omega_{g} M$, so

$$
\begin{aligned}
\Omega_{g} & =\left(\mathbb{I}_{2 g}+p \cdot A\right)^{t} \Omega_{g}\left(\mathbb{I}_{2 g}+p \cdot A\right) \\
& =\Omega_{g}+p \cdot\left(A^{t} \Omega_{g}+\Omega_{g} A\right)+p^{2} A^{t} \Omega_{g} A .
\end{aligned}
$$

This implies that modulo $p$, we have $A^{t} \Omega_{g}+\Omega_{g} A=0$. Next, we prove that $\psi$ is a homomorphism. Consider $M, N \in \mathrm{Sp}_{2 g}(\mathbb{Z}, p)$ with $M=\mathbb{I}_{2 g}+p A$ and $N=\mathbb{I}_{2 g}+p B$. We then have $M N=\mathbb{I}_{2 g}+p(A+B)+p^{2} A B$, so modulo $p$ we have $\psi(M N)=A+B$.

It remains to show that $\psi$ is surjective. If $p$ is prime, then this is easy. Indeed, $\psi$ is equivariant with respect to the conjugation actions of $\operatorname{Sp}_{2 g}(\mathbb{Z})$ on $\operatorname{Sp}_{2 g}(\mathbb{Z}, p)$ and $\mathfrak{s p}_{2 g}(\mathbb{Z} / p)$. The latter factors through $\operatorname{Sp}_{2 g}(\mathbb{Z} / p)$, so the image of $\psi$ is a $\operatorname{Sp}_{2 g}(\mathbb{Z} / p)$ subrepresentation of $\mathfrak{s p}_{2 g}(\mathbb{Z} / p)$. However, it is well-known that $\mathfrak{s p}_{2 g}(\mathbb{Z} / p)$ is an irreducible $\operatorname{Sp}_{2 g}(\mathbb{Z} / p)$-representation, so since $\psi$ is not trivial it must be surjective (we remark that this uses the fact that $p$ is odd - the abelian group $\mathfrak{s p}_{2 g}(\mathbb{Z} / 2)$ is not an irreducible representation of $\left.\mathrm{Sp}_{2 g}(\mathbb{Z} / 2)\right)$. We leave the general case as an exercise.

Exercise 3.7. Prove that $\psi$ is surjective by constructing elements of $\operatorname{Sp}_{2 g}(\mathbb{Z}, p)$ that map to generators of $\mathfrak{s p}_{2 g}(\mathbb{Z} / p)$.

Finishing the proof of Theorem 3.6. Observe now that $\operatorname{ker}(\psi)=\operatorname{Sp}_{2 g}\left(\mathbb{Z}, p^{2}\right)$. Since the target of $\psi$ is abelian, this implies that

$$
\left[\mathrm{Sp}_{2 g}(\mathbb{Z}, p), \mathrm{Sp}_{2 g}(\mathbb{Z}, p)\right] \subset \mathrm{Sp}_{2 g}\left(\mathbb{Z}, p^{2}\right) .
$$

Theorem 3.6 thus follows from the following lemma.

Lemma 3.8. If $g, p \geq 3$ and $p$ is odd, then $\operatorname{Sp}_{2 g}\left(\mathbb{Z}, p^{2}\right) \subset\left[\operatorname{Sp}_{2 g}(\mathbb{Z}, p), \operatorname{Sp}_{2 g}(\mathbb{Z}, p)\right]$.

The proof of Lemma 3.8 follows from a direct matrix calculation. Rather than giving the details (which can be found in [33, Lemma 2.4] or [39, Proposition 10.1]), we will give an exercise which outlines a proof of a somewhat easier result for $\mathrm{SL}_{n}(\mathbb{Z})$. The proof of Lemma 3.8 follows the same basic pattern, though the details are more complicated.

Exercise 3.9. Let $\mathrm{SL}_{n}(\mathbb{Z}, p)$ be the kernel of the natural map $\mathrm{SL}_{n}(\mathbb{Z}) \rightarrow \mathrm{SL}_{n}(\mathbb{Z} / p)$. In this exercise, you will prove a theorem of Lee and Szczarba [26] which says that for $n \geq 3$, we have $\mathrm{H}_{1}\left(\mathrm{SL}_{n}(\mathbb{Z}, p) ; \mathbb{Z}\right) \cong \mathfrak{s l}_{n}(\mathbb{Z} / p)$. Here $\mathfrak{s l}_{n}(\mathbb{Z} / p)$ is the abelian group of $n \times n$ matrices over $\mathbb{Z} / p$ with trace 0 . We remark that in this exercise, we do not need to assume that $p$ is odd.

1. Construct a surjective homomorphism $\mathrm{SL}_{n}(\mathbb{Z}, p) \rightarrow \mathfrak{s l}_{n}(\mathbb{Z} / p)$ whose kernel is $\mathrm{SL}_{n}\left(\mathbb{Z} / p^{2}\right)$. Conclude that $\left[\mathrm{SL}_{n}(\mathbb{Z}, p), \mathrm{SL}_{n}(\mathbb{Z}, p)\right] \subset \mathrm{SL}_{n}\left(\mathbb{Z}, p^{2}\right)$. 
2. Prove that $\mathrm{SL}_{n}\left(\mathbb{Z} / p^{2}\right) \subset\left[\mathrm{SL}_{n}(\mathbb{Z}, p), \mathrm{SL}_{n}(\mathbb{Z}, p)\right]$. You will need the following theorem of Bass, Milnor, and Serre [1]. For $1 \leq i, j \leq n$ such that $i \neq j$, let $e_{i j}$ be the elementary matrix which is obtained from the $n \times n$ identity matrix by changing the entry at position $(i, j)$ to 1 . Bass, Milnor, and Serre proved that for $n \geq 3$ and $q \geq 2$, the group $\mathrm{SL}_{n}(\mathbb{Z}, q)$ is normally generated (as a subgroup of $\left.\mathrm{SL}_{n}(\mathbb{Z})\right)$ by the set $\left\{e_{i j}^{q} \mid 1 \leq i, j \leq n, i \neq j\right\}$.

\section{Lecture 4 : The second rational homology group of $\operatorname{Mod}_{g}(p)$}

In this final lecture, we turn to the second homology group of $\operatorname{Mod}_{g}(p)$. To set the stage, let us first recall what happens for the first homology group. Powell [31] proved that $\mathrm{H}_{1}\left(\operatorname{Mod}_{g} ; \mathbb{Z}\right)=0$ for $g \geq 3$. See $[12, \S 5.1]$ for an easier proof (due to Harer). Hain [13] later proved that $\mathrm{H}_{1}\left(\operatorname{Mod}_{g}(p) ; \mathbb{Q}\right)=0$ for $g \geq 3$. In other words, over $\mathbb{Q}$ the first homology group of $\operatorname{Mod}_{g}$ does not change when you pass to the finiteindex subgroup $\operatorname{Mod}_{g}(p)$. Our proof of Theorem 3.1 in Lecture 3 is essentially an elaboration of Hain's proof.

Harer [14] proved that $\mathrm{H}_{2}\left(\operatorname{Mod}_{g} ; \mathbb{Z}\right) \cong \mathbb{Z}$ for $g \geq 4$. The following theorem says that a similar result holds for $\operatorname{Mod}_{g}(p)$ as long as we work over $\mathbb{Q}$.

Theorem 4.1 (Putman, [37]). For $g \geq 5$ and $p \geq 2$, we have $\mathrm{H}_{2}\left(\operatorname{Mod}_{g}(p) ; \mathbb{Q}\right) \cong \mathbb{Q}$.

The first part of this lecture is devoted to motivating why one might expect such a theorem to hold. Next, we show that Theorem 4.1 is equivalent to a weak form of homological stability for $\operatorname{Mod}_{g}(p)$. Finally, we discuss how to prove this stability result.

The transfer map. What happens to the homology of a group when you pass to a finite-index subgroup? The following standard lemma says that over $\mathbb{Q}$, the homology can get larger but it cannot get smaller.

Lemma 4.2. If $G$ is a finite-index subgroup of $\Gamma$, then the map $\mathrm{H}_{k}(G ; \mathbb{Q}) \rightarrow \mathrm{H}_{k}(\Gamma ; \mathbb{Q})$ is surjective for all $k \geq 1$.

Example. If $\Gamma$ is a free abelian group and $G$ is a finite-index subgroup of $\Gamma$, then there exists some $\ell \geq 1$ such that $\ell \cdot \Gamma<G<\Gamma$. Consequently, we have $G \cong \Gamma$ and it is easy to see that the map $\mathrm{H}_{k}(G ; \mathbb{Q}) \rightarrow \mathrm{H}_{k}(\Gamma ; \mathbb{Q})$ is an isomorphism for all $k \geq 1$.

Example. If $\Gamma$ is a free group of rank $n$ and $G$ is a proper finite-index subgroup of $\Gamma$, then $G$ is a free group of rank strictly greater than $n$. Consequently, the rank of $\mathrm{H}_{1}(G ; \mathbb{Q})$ is strictly greater than the rank of $\mathrm{H}_{1}(\Gamma ; \mathbb{Q}) \cong \mathbb{Q}^{n}$.

Remark. Lemma 4.2 is false over $\mathbb{Z}$. Indeed, if $\Gamma$ is a finite group, then $G=1$ is a finite-index subgroup of $\Gamma$. The homology groups of $G$ are all trivial, but $\Gamma$ can certainly have nontrivial homology groups. However, Lemma 4.2 does imply that the homology groups of $\Gamma$ are all torsion.

Proof of Lemma 4.2. Let $n=[\Gamma: G]$. The key to the proof is the transfer map. Letting $R$ be a commutative ring and letting $i_{*}: \mathrm{H}_{k}(G ; R) \rightarrow \mathrm{H}_{k}(\Gamma ; R)$ be the 
map induced by the inclusion $i: G \hookrightarrow \Gamma$, the transfer map is a homomorphism $\tau_{k}: \mathrm{H}_{k}(\Gamma ; R) \rightarrow \mathrm{H}_{k}(G ; R)$ satisfying

$$
i_{*}\left(\tau_{k}(x)\right)=n x \quad \text { for all } x \in \mathrm{H}_{k}(\Gamma ; R) .
$$

The existence of $\tau_{k}$ immediately implies the lemma. Indeed, if $R=\mathbb{Q}$, then $\frac{1}{n} \tau_{k}$ is a right-inverse to $i_{*}$, so $i_{*}$ is surjective.

The transfer map is not induced by a homomorphism $\Gamma \rightarrow G$; rather, it is constructed at the level of chains. The construction goes as follows. Let $B \Gamma$ be a $K(\Gamma, 1)$ and let $\rho: B G \rightarrow B \Gamma$ be the cover corresponding to $G<\Gamma$. Thus $B G$ is a $K(G, 1)$ and $\rho$ is a degree $n$ cover. Define a map $\hat{\tau}_{k}: C_{k}(B \Gamma ; R) \rightarrow C_{k}(B G ; R)$ as follows. If $\sigma^{k} \in C_{k}(B \Gamma ; R)$ is a singular $k$-simplex, then there are exactly $n$ singular $k$-simplices $\tilde{\sigma}_{1}^{k}, \ldots, \tilde{\sigma}_{n}^{k}$ in $B G$ satisfying $\rho_{*}\left(\tilde{\sigma}_{i}^{k}\right)=\sigma^{k}$. Define

$$
\hat{\tau}_{k}\left(\sigma^{k}\right)=\tilde{\sigma}_{1}^{k}+\cdots+\tilde{\sigma}_{n}^{k} .
$$

Exercise 4.3. The maps $\hat{\tau}_{k}$ commute with the boundary operators, so we have a commutative diagram

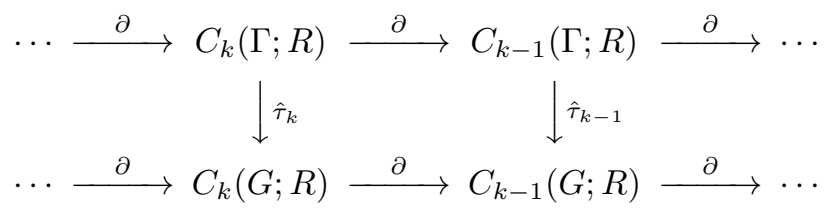

By this exercise, the maps $\hat{\tau}_{k}$ induce maps $\tau_{k}: \mathrm{H}_{k}(\Gamma ; R) \rightarrow \mathrm{H}_{k}(G ; R)$ in homology. Clearly the maps $\tau_{k}$ satisfy (5), so we are done.

Borel stability. Lemma 4.2 implies that $\mathrm{H}_{2}\left(\operatorname{Mod}_{g}(p) ; \mathbb{Q}\right) \cong \mathbb{Q}^{m}$ for some $m \geq 1$. Why should we expect that $m=1$ ? There is a very fruitful analogy between the mapping class group and lattices in Lie groups. The Borel stability theorem says that for the classical sequences of arithmetic lattices, passing to finite-index subgroups does not change their rational homology in a stable range. For example, denote $\mathrm{SL}_{n}(\mathbb{Z})$ by $\Gamma_{n}$ and define

$$
\Gamma_{n}(p)=\operatorname{ker}\left(\mathrm{SL}_{n}(\mathbb{Z}) \rightarrow \mathrm{SL}_{n}(\mathbb{Z} / p)\right)
$$

for $p \geq 2$. Here the map $\mathrm{SL}_{n}(\mathbb{Z}) \rightarrow \mathrm{SL}_{n}(\mathbb{Z} / p)$ comes from reducing all the entries in the matrices modulo $p$. Borel's theorem then takes the following form.

Theorem 4.4 (Borel, [6]). For $k \geq 1$, there exists some $N_{k} \geq 1$ such that if $n \geq N_{k}$ and $p \geq 2$, then $\mathrm{H}_{k}\left(\Gamma_{n}(p) ; \mathbb{Q}\right) \cong \mathrm{H}_{k}\left(\Gamma_{n} ; \mathbb{Q}\right)$.

One can view Theorem 4.1 as an analogue (for $k=2$ ) of Theorem 4.4 for the mapping class group. As we mentioned, Hain [13] proved a similar theorem for $k=1$. It would be very interesting to extend this to $k \geq 3$.

Reduction to stability. We now discuss the proof of Theorem 4.1. In [15], Harer proved that the homology groups of $\operatorname{Mod}_{g}$ satisfy homological stability. See [18] or [40] for more readable proofs of this. One special case of Harer's theorem is 


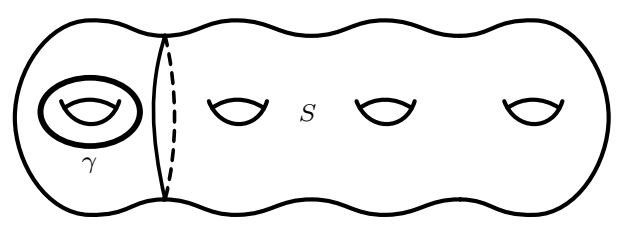

Figure 4.1: $S$ is a subsurface of $\Sigma_{g}$ satisfying $S \cong \Sigma_{g-1,1}$ and $\gamma$ is a simple closed nonseparating curve contained in $\Sigma_{g} \backslash S$

as follows. Choose a subsurface $S$ of $\Sigma_{g}$ such that $S \cong \Sigma_{g-1,1}$ (see Figure 4.1). Extending mapping classes on $S$ by the identity to $\Sigma_{g}$, we get a homomorphism $\operatorname{Mod}_{g-1,1} \rightarrow \operatorname{Mod}_{g}$. Harer proved that for each $k$, there exists some $N_{k}$ such that if $g \geq N_{k}$, then the induced map $\mathrm{H}_{k}\left(\operatorname{Mod}_{g-1,1} ; \mathbb{Z}\right) \rightarrow \mathrm{H}_{k}\left(\operatorname{Mod}_{g} ; \mathbb{Z}\right)$ is an isomorphism.

The following result asserts that something similar happens for $\operatorname{Mod}_{g}(p)$ for $k=2$.

Lemma 4.5. For $g \geq 5$ and $p \geq 2$, let $i: \operatorname{Mod}_{g-1,1}(p) \rightarrow \operatorname{Mod}_{g}(p)$ be the restriction of the map $\operatorname{Mod}_{g-1,1} \rightarrow \operatorname{Mod}_{g}$ described above. Then the induced map $i_{*}: \mathrm{H}_{2}\left(\operatorname{Mod}_{g-1,1}(p) ; \mathbb{Q}\right) \rightarrow \mathrm{H}_{2}\left(\operatorname{Mod}_{g}(p) ; \mathbb{Q}\right)$ is surjective.

Observe that Theorem 4.1 implies Lemma 4.5. Indeed, if Theorem 4.1 is true, then we have a commutative diagram of the form

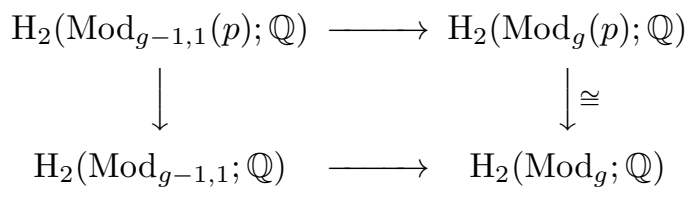

By Lemma 4.2, the map $\mathrm{H}_{2}\left(\operatorname{Mod}_{g-1,1}(p) ; \mathbb{Q}\right) \rightarrow \mathrm{H}_{2}\left(\operatorname{Mod}_{g-1,1} ; \mathbb{Q}\right)$ is a surjection, and Harer's stability theorem say that the map $\mathrm{H}_{2}\left(\operatorname{Mod}_{g-1,1} ; \mathbb{Q}\right) \rightarrow \mathrm{H}_{2}\left(\operatorname{Mod}_{g} ; \mathbb{Q}\right)$ is an isomorphism as long as $g \geq 5$. We conclude that the map $\mathrm{H}_{2}\left(\operatorname{Mod}_{g-1,1}(p) ; \mathbb{Q}\right) \rightarrow$ $\mathrm{H}_{2}\left(\operatorname{Mod}_{g}(p) ; \mathbb{Q}\right)$ is a surjection, as desired.

Somewhat surprisingly, Lemma 4.5 also implies Theorem 4.1.

Lemma 4.6. Lemma 4.5 implies Theorem 4.1.

Before proving Lemma 4.6, we need some more abstract nonsense. Let $G$ be a finiteindex normal subgroup of $\Gamma$. The conjugation action of $\Gamma$ on $G$ induces an action of $\Gamma$ on $\mathrm{H}_{k}(G ; \mathbb{Q})$. Recall that if $M$ is a vector space upon which $\Gamma$ acts, then the coinvariants of the action, denoted $M_{\Gamma}$, is the quotient $M / I$ with $I$ the subspace spanned by $\{x-g(x) \mid x \in M, g \in \Gamma\}$. In other words, $M_{\Gamma}$ is the largest quotient of $M$ upon which $\Gamma$ acts trivially. Since $\Gamma$ acts trivially on its own homology groups $\mathrm{H}_{k}(\Gamma ; \mathbb{Q})$, the map $\mathrm{H}_{k}(G ; \mathbb{Q}) \rightarrow \mathrm{H}_{k}(\Gamma ; \mathbb{Q})$ factors through $\left(\mathrm{H}_{k}(G ; \mathbb{Q})\right)_{\Gamma}$. We then have the following exercise.

Exercise 4.7. $\mathrm{H}_{k}(\Gamma ; \mathbb{Q}) \cong\left(\mathrm{H}_{k}(G ; \mathbb{Q})\right)_{\Gamma}$. Hint : carefully study the proof of Lemma 4.2. Alternatively, this can be proven using the Hochschild-Serre spectral sequence of the extension

$$
1 \longrightarrow G \longrightarrow \Gamma \longrightarrow \Gamma / G \longrightarrow 1
$$


Proof of Lemma 4.6. Assume that Lemma 4.5 holds. Since $\mathrm{H}_{2}\left(\operatorname{Mod}_{g} ; \mathbb{Q}\right) \cong \mathbb{Q}$, to prove that $\mathrm{H}_{2}\left(\operatorname{Mod}_{g}(p) ; \mathbb{Q}\right) \cong \mathbb{Q}$, it is enough to prove that $\mathrm{H}_{2}\left(\operatorname{Mod}_{g}(p) ; \mathbb{Q}\right) \cong$ $\mathrm{H}_{2}\left(\operatorname{Mod}_{g} ; \mathbb{Q}\right)$. By Exercise 4.7 , this is equivalent to showing that the action of $\operatorname{Mod}_{g}$ on $\mathrm{H}_{2}\left(\operatorname{Mod}_{g}(p) ; \mathbb{Q}\right)$ is trivial. This can be checked on a generating set. The group $\operatorname{Mod}_{g}$ is generated by the set of Dehn twists about nonseparating simple closed curves. Consider such a Dehn twist $T_{\gamma}$. As is shown in Figure 4.1, we can find a subsurface $S$ of $\Sigma_{g}$ such that $S \cong \Sigma_{g-1,1}$ and such that $\gamma \subset \Sigma_{g} \backslash S$. Let $i: \operatorname{Mod}_{g-1,1}(p) \rightarrow \operatorname{Mod}_{g}(p)$ be the map induced by the subsurface inclusion $S \hookrightarrow \Sigma_{g}$. Clearly $T_{\gamma}$ commutes with $\operatorname{Im}(i)$, so $T_{\gamma}$ acts trivially on $i_{*}\left(\mathrm{H}_{2}\left(\operatorname{Mod}_{g-1,1}(p) ; \mathbb{Q}\right)\right)$. Lemma 4.5 implies that $i_{*}\left(\mathrm{H}_{2}\left(\operatorname{Mod}_{g-1,1}(p) ; \mathbb{Q}\right)\right)=\mathrm{H}_{2}\left(\operatorname{Mod}_{g}(p) ; \mathbb{Q}\right)$, so we conclude that $T_{\gamma}$ acts trivially on $\mathrm{H}_{2}\left(\operatorname{Mod}_{g}(p) ; \mathbb{Q}\right)$, as desired.

A weaker result suffices. In fact, we do not need the full strength of Lemma 4.5 to prove Theorem 4.1. If $\gamma$ is the isotopy class of a nonseparating simple closed curve on $\Sigma_{g}$, then denote by $\left(\operatorname{Mod}_{g}(p)\right)_{\gamma}$ the subgroup of $\operatorname{Mod}_{g}(p)$ which stabilizes $\gamma$. Examining the proof of Lemma 4.6, it is clear that the following lemma also implies Theorem 4.1.

Lemma 4.8. For $g \geq 5$ and $p \geq 2$, let $\gamma$ be a nonseparating simple closed curve on $\Sigma_{g}$. Then the map $\mathrm{H}_{2}\left(\left(\operatorname{Mod}_{g}(p)\right)_{\gamma} ; \mathbb{Q}\right) \rightarrow \mathrm{H}_{2}\left(\operatorname{Mod}_{g}(p) ; \mathbb{Q}\right)$ is surjective.

The homological stability machine. Homological stability theorems are known for many different sequences of groups, and there is now a standard procedure for proving them. This procedure goes back to Quillen. See [17] for an excellent discussion of how this machine applies to many different sequences of groups and [40] for a detailed exposition of how to apply it to the mapping class group. The input for this machine consists of a sequence of highly connected spaces upon which the groups in question act. For $\operatorname{Mod}_{g}$, Harer proved homological stability by studying the action on the following space.

Definition. The nonseparating curve complex, denoted $\mathcal{C}_{g}^{\text {ns }}$, is the simplicial complex whose $k$-simplices are sets $\left\{\gamma_{0}, \ldots, \gamma_{k}\right\}$ of isotopy classes of simple closed curves on $\Sigma_{g}$ that can be realized such that the $\gamma_{i}$ are all disjoint and $\Sigma_{g} \backslash\left(\gamma_{0} \cup \cdots \cup \gamma_{k}\right)$ is connected.

One's first impulse, of course, is to apply this machine to $\operatorname{Mod}_{g}(p)$ to try to prove Lemma 4.8. Unfortunately, it does not quite work. The problem is that if it did work, then it would prove that $\mathrm{H}_{k}\left(\operatorname{Mod}_{g}(p) ; \mathbb{Z}\right)$ is stable, and this is false even for $k=1$ (see Theorem 3.1). The machine does, however, give the following result.

Lemma 4.9. For $g \geq 5$ and $p \geq 2$, the map

$$
\bigoplus_{\gamma \in\left(\mathcal{C}_{g}^{n s}\right)(0)} \mathrm{H}_{2}\left(\left(\operatorname{Mod}_{g}(p)\right)_{\gamma} ; \mathbb{Q}\right) \longrightarrow \mathrm{H}_{2}\left(\operatorname{Mod}_{g}(p) ; \mathbb{Q}\right)
$$

is surjective. 
Rescuing the machine. To deduce Lemma 4.8 from Lemma 4.9, we need to prove that if $g \geq 5$ and $p \geq 2$, then for any two nonseparating simple closed curves $\gamma$ and $\gamma^{\prime}$ on $\Sigma_{g}$, the images of $\mathrm{H}_{2}\left(\left(\operatorname{Mod}_{g}(p)\right)_{\gamma} ; \mathbb{Q}\right)$ and $\mathrm{H}_{2}\left(\left(\operatorname{Mod}_{g}(p)\right)_{\gamma^{\prime}} ; \mathbb{Q}\right)$ in $\mathrm{H}_{2}\left(\operatorname{Mod}_{g}(p) ; \mathbb{Q}\right)$ are the same. A standard result about surfaces called the "change of coordinates principle" (see [12]) says that there exists some $\phi \in \operatorname{Mod}_{g}$ such that $\phi(\gamma)=\gamma^{\prime}$. This implies that

$$
\phi\left(\operatorname{Mod}_{g}(p)\right)_{\gamma} \phi^{-1}=\left(\operatorname{Mod}_{g}(p)\right)_{\gamma^{\prime}} .
$$

Thus the action of $\operatorname{Mod}_{g}$ on $\mathrm{H}_{2}\left(\operatorname{Mod}_{g}(p) ; \mathbb{Q}\right)$ takes the image of $\mathrm{H}_{2}\left(\left(\operatorname{Mod}_{g}(p)\right)_{\gamma} ; \mathbb{Q}\right)$ to the image of $\mathrm{H}_{2}\left(\left(\operatorname{Mod}_{g}(p)\right)_{\gamma^{\prime}} ; \mathbb{Q}\right)$. We conclude that Lemma 4.9 would following from the following lemma.

Lemma 4.10. For $g \geq 5$ and $p \geq 2$, if $\gamma$ is a nonseparating simple closed curve on $\Sigma_{g}$, then $\operatorname{Mod}_{g}$ acts trivially on the image of $\mathrm{H}_{2}\left(\left(\operatorname{Mod}_{g}(p)\right)_{\gamma} ; \mathbb{Q}\right)$ in $\mathrm{H}_{2}\left(\operatorname{Mod}_{g}(p) ; \mathbb{Q}\right)$.

The proof of Lemma 4.10 is quite complicated (see [37]). Rather than give it here, we will prove an analogous result about $\Gamma_{n}=\mathrm{SL}_{n}(\mathbb{Z})$. Recall that

$$
\Gamma_{n}(p)=\operatorname{ker}\left(\mathrm{SL}_{n}(\mathbb{Z}) \longrightarrow \mathrm{SL}_{n}(\mathbb{Z} / p)\right)
$$

There is a natural map $\Gamma_{n-1} \rightarrow \Gamma_{n}$ which takes a matrix $A \in \Gamma_{n-1}$ to the matrix $\left(\begin{array}{ll}1 & 0 \\ 0 & A\end{array}\right) \in \Gamma_{n}$. In [9], Charney proves the following theorem.

Theorem 4.11 (Charney, [9, Proposition 5.5]). For $n \geq 2$ and $k, p \geq 1$, the group $\Gamma_{n}$ acts trivially on the image of $\mathrm{H}_{k}\left(\Gamma_{n-1}(p) ; \mathbb{Q}\right)$ in $\mathrm{H}_{k}\left(\Gamma_{n}(p) ; \mathbb{Q}\right)$.

Though she does not say it in her paper, it is not hard to combine this with the standard homological stability machinery to give a new proof of the Borel stability theorem for $\mathrm{SL}_{n}(\mathbb{Z})$. After the proof, we will comment on the relation between the proofs of Theorem 4.11 and Lemma 4.10 .

Proof of Theorem 4.11. For $1 \leq i, j \leq n$ such that $i \neq j$, let $e_{i j}$ be the elementary matrix which is obtained from the $n \times n$ identity matrix by changing the entry at position $(i, j)$ to 1 . The group $\Gamma_{n}$ is generated by the set $\left\{e_{i j} \mid 1 \leq i, j \leq n, i \neq j\right\}$. However, we do not need this entire set.

Exercise 4.12. For $2 \leq i, j \leq n$ such that $i \neq j$, prove that $\left[e_{i 1}, e_{1 j}\right]=e_{i j}$.

Consequently, we only need to prove that $e_{1 j}$ and $e_{i 1}$ act trivially for $2 \leq i, j \leq n$. We will give the details for $e_{1 j}$. The other case is similar.

Consider the subgroups

$$
G=\left\{\left(\begin{array}{c|cc}
1 & c_{2} \cdots c_{n} \\
\hline 0 & \\
\vdots & A \\
0 &
\end{array}\right) \mid A \in \Gamma_{n-1}(p), c_{2}, \ldots, c_{n} \in p \mathbb{Z}\right\}
$$


and

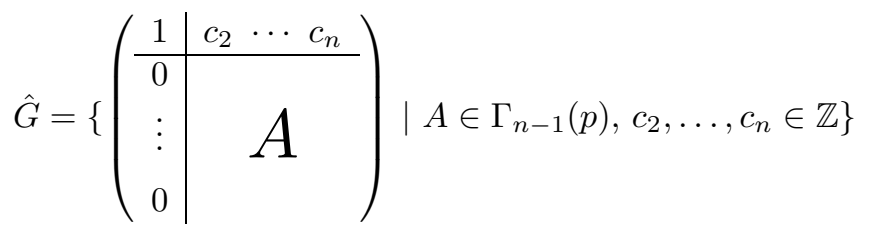

of $\Gamma_{n}$. The conjugation action of $e_{1 j}$ on $\Gamma_{n}$ takes $G$ and $\hat{G}$ to themselves. We have $\Gamma_{n-1}(p) \subset G \subset \Gamma_{n}(p)$, so it is enough to show that $e_{1 j}$ acts trivially on $\mathrm{H}_{k}(G ; \mathbb{Q})$. Since $e_{1 j} \in \hat{G}$, the action of $e_{1 j}$ on $\mathrm{H}_{k}(\hat{G} ; \mathbb{Q})$ is trivial. It is therefore enough to show that the inclusion $G \hookrightarrow \hat{G}$ induces an isomorphism $\mathrm{H}_{k}(G ; \mathbb{Q}) \cong \mathrm{H}_{k}(\hat{G} ; \mathbb{Q})$.

Define

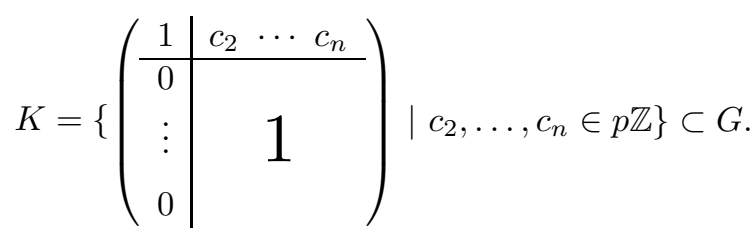

Observe that $K \cong \mathbb{Z}^{n-1}$. We have a short exact sequence

$$
1 \longrightarrow K \longrightarrow G \longrightarrow \Gamma_{n-1}(p) \longrightarrow 1
$$

Similarly, setting

$$
\hat{K}=\left\{\left(\begin{array}{c|cc}
1 & c_{2} \cdots & c_{n} \\
\hline 0 & \\
\vdots & 1 \\
0 &
\end{array}\right) \mid c_{2}, \ldots, c_{n} \in \mathbb{Z}\right\} \subset \hat{G}
$$

we have a short exact sequence

$$
1 \longrightarrow \hat{K} \longrightarrow \hat{G} \longrightarrow \Gamma_{n-1}(p) \longrightarrow 1 .
$$

These short exact sequences fit into a commutative diagram of the form

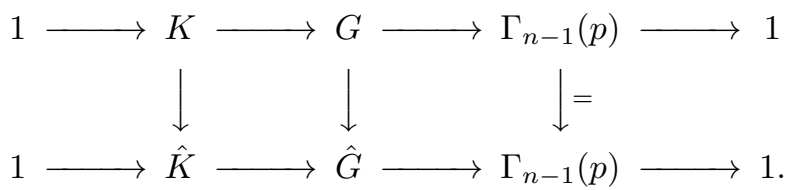

By Lemma 4.13 (a sort of 5-lemma for group homology), it is enough to show that the inclusion map $K \hookrightarrow \hat{K}$ induces an isomorphism $\mathrm{H}_{k}(K ; \mathbb{Q}) \cong \mathrm{H}_{k}(\hat{K} ; \mathbb{Q})$. However, we have $\hat{K} \cong \mathbb{Z}^{n-1}$ and $K=p \cdot \hat{K}$, so this is immediate. We remark that this final step is the only place we use the fact that we are working over $\mathbb{Q}$. 
Lemma 4.13 (5-lemma for group homology). Fix a commutative ring $R$. Consider a commutative diagram

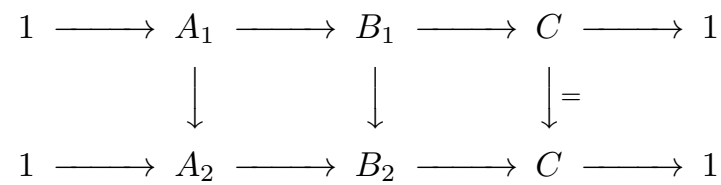

of short exact sequences of groups. Assume that the map $A_{1} \rightarrow A_{2}$ induces an isomorphism $\mathrm{H}_{k}\left(A_{1} ; R\right) \cong \mathrm{H}_{k}\left(A_{2} ; R\right)$ for all $k$. Then the map $B_{1} \rightarrow B_{2}$ induces an isomorphism $\mathrm{H}_{k}\left(B_{1} ; R\right) \cong \mathrm{H}_{k}\left(B_{2} ; R\right)$ for all $k$.

Proof. Associated each of our short exact sequences is a Hochschild-Serre spectral sequence in group homology. The assumptions in the lemma imply that the induced map between these spectral sequences is an isomorphism on the $E^{2}$-page. This implies that it converges an an isomorphism on the $E^{\infty}$-pages, so we obtain isomorphisms $\mathrm{H}_{k}\left(B_{1} ; R\right) \cong \mathrm{H}_{k}\left(B_{2} ; R\right)$ for all $k$.

Relation between Theorem 4.11 and Lemma 4.10. The proof of Lemma 4.10 in [37] is not nearly as short as the proof above of Theorem 4.11. However, they share some features. The key to the proof of Theorem 4.11 above is the short exact sequence

$$
1 \longrightarrow K \longrightarrow G \longrightarrow \Gamma_{n-1}(p) \longrightarrow 1 \text {. }
$$

A similar role in the proof of Lemma 4.10 is played by various analogues for $\operatorname{Mod}_{g}(p)$ of the Birman exact sequence which was discussed in Lecture 1. However, the kernels of these Birman exact sequences are not free abelian, so passing to finite-index subgroups actually changes their isomorphism types. This necessitates making a number of rather intricate twisted group cohomology computations.

Recap. The above proof that $\mathrm{H}_{2}\left(\operatorname{Mod}_{g}(p) ; \mathbb{Q}\right) \cong \mathbb{Q}$ was a little involved, so let us recap the main steps.

1. We first showed that $\mathrm{H}_{2}\left(\operatorname{Mod}_{g}(p) ; \mathbb{Q}\right) \cong \mathbb{Q}$ if and only if $\mathrm{H}_{2}\left(\operatorname{Mod}_{g}(p) ; \mathbb{Q}\right)$ satisfies a weak form of homological stability (see Lemma 4.6).

2. We then attempted to use the standard homological stability machinery to prove that $\mathrm{H}_{2}\left(\operatorname{Mod}_{g}(p) ; \mathbb{Q}\right)$ stabilizes. This failed, but it showed that $\mathrm{H}_{2}\left(\operatorname{Mod}_{g}(p) ; \mathbb{Q}\right)$ is "concentrated" on stabilizers of simple closed curves (see Lemma 4.9).

3. The proof is reduced to showing that all stabilizers of simple closed curves give the same "chunk" of $\mathrm{H}_{2}\left(\operatorname{Mod}_{g}(p) ; \mathbb{Q}\right)$.

It seems reasonable to conjecture that $\mathrm{H}_{k}\left(\operatorname{Mod}_{g}(p) ; \mathbb{Q}\right) \cong \mathrm{H}_{k}\left(\operatorname{Mod}_{g} ; \mathbb{Q}\right)$ in a stable range for all $k$. The first two steps above can be easily generalized to $\mathrm{H}_{k}\left(\operatorname{Mod}_{g}(p) ; \mathbb{Q}\right)$ for $k \geq 3$. The real difficulty is the third step. It would be very interesting to extend it to the higher homology groups. 


\section{References}

[1] H. Bass, J. Milnor and J.-P. Serre, Solution of the congruence subgroup problem for $\mathrm{SL}_{n}(n \geq 3)$ and $\operatorname{Sp}_{2 n}(n \geq 2)$, Inst. Hautes Études Sci. Publ. Math. No. 33 (1967), 59-137.

[2] J. S. Birman, Mapping class groups and their relationship to braid groups, Comm. Pure Appl. Math. 22 (1969), 213-238.

[3] J. S. Birman, On Siegel's modular group, Math. Ann. 191 (1971), 59-68.

[4] J. S. Birman and R. Craggs, The $\mu$-invariant of 3-manifolds and certain structural properties of the group of homeomorphisms of a closed, oriented 2-manifold, Trans. Amer. Math. Soc. 237 (1978), 283-309.

[5] S. Boldsen, Improved homological stability for the mapping class group with integral or twisted coefficients, preprint 2009.

[6] A. Borel, Stable real cohomology of arithmetic groups, Ann. Sci. École Norm. Sup. (4) 7 (1974), 235-272 (1975).

[7] N. Broaddus, B. Farb and A. Putman, Irreducible Sp-representations and subgroup distortion in the mapping class group, Comment. Math. Helv. 86 (2011), 537-556.

[8] K. S. Brown, Cohomology of groups, corrected reprint of the 1982 original, Graduate Texts in Mathematics, 87, Springer, New York, 1994.

[9] R. Charney, On the problem of homology stability for congruence subgroups, Comm. Algebra 12 (1984), no. 17-18, 2081-2123.

[10] T. Church and B. Farb, Higher period maps, a question of Johnson, and a homological stability conjecture for the Torelli group, preprint 2009.

[11] D. B. A. Epstein, Curves on 2-manifolds and isotopies, Acta Math. 115 (1966), 83-107.

[12] B. Farb and D. Margalit, A primer on mapping class groups, to be published by Princeton University Press.

[13] R. Hain, Torelli groups and geometry of moduli spaces of curves, in Current topics in complex algebraic geometry (Berkeley, CA, 1992/93), 97-143, Cambridge Univ. Press, Cambridge.

[14] J. Harer, The second homology group of the mapping class group of an orientable surface, Invent. Math. 72 (1983), no. 2, 221-239.

[15] J. L. Harer, Stability of the homology of the mapping class groups of orientable surfaces, Ann. of Math. (2) 121 (1985), no. 2, 215-249.

[16] A. Hatcher and D. Margalit, Generating the Torelli group, in preparation.

[17] A. Hatcher and N. Wahl, Stabilization for mapping class groups of 3-manifolds, Duke Math. J. 155 (2010), no. 2, 205-269.

[18] N. V. Ivanov, Complexes of curves and Teichmüller modular groups, Uspekhi Mat. Nauk 42 (1987), no. 3(255), 49-91

[19] D. Johnson, Homeomorphisms of a surface which act trivially on homology, Proc. Amer. Math. Soc. 75 (1979), no. 1, 119-125.

[20] D. Johnson, An abelian quotient of the mapping class group $\mathcal{I}_{g}$, Math. Ann. 249 (1980), no. $3,225-242$.

[21] D. Johnson, Quadratic forms and the Birman-Craggs homomorphisms, Trans. Amer. Math. Soc. 261 (1980), no. 1, 235-254. 
[22] D. Johnson, The structure of the Torelli group. I. A finite set of generators for $\mathcal{I}$, Ann. of Math. (2) 118 (1983), no. 3, 423-442.

[23] D. Johnson, The structure of the Torelli group. II. A characterization of the group generated by twists on bounding curves, Topology 24 (1985), no. 2, 113-126.

[24] D. Johnson, The structure of the Torelli group. III. The abelianization of $\mathcal{T}$, Topology 24 (1985), no. 2, 127-144.

[25] D. Johnson, A survey of the Torelli group, in Low-dimensional topology (San Francisco, Calif., 1981), 165-179, Contemp. Math., 20, Amer. Math. Soc., Providence, RI.

[26] R. Lee and R. H. Szczarba, On the homology and cohomology of congruence subgroups, Invent. Math. 33 (1976), no. 1, 15-53.

[27] I. Madsen and M. Weiss, The stable moduli space of Riemann surfaces: Mumford's conjecture, Ann. of Math. (2) 165 (2007), no. 3, 843-941.

[28] D. McCullough and A. Miller, The genus 2 Torelli group is not finitely generated, Topology Appl. 22 (1986), no. 1, 43-49.

[29] G. Mess, The Torelli groups for genus 2 and 3 surfaces, Topology 31 (1992), no. 4, 775-790.

[30] B. Perron, Filtration de Johnson et groupe de Torelli modulo $p, p$ premier, C. R. Math. Acad. Sci. Paris 346 (2008), no. 11-12, 667-670.

[31] J. Powell, Two theorems on the mapping class group of a surface, Proc. Amer. Math. Soc. 68 (1978), no. 3, 347-350.

[32] A. Putman, Cutting and pasting in the Torelli group, Geom. Topol. 11 (2007), 829-865.

[33] A. Putman The abelianization of the level L mapping class group, preprint 2008.

[34] A. Putman, An infinite presentation of the Torelli group, Geom. Funct. Anal. 19 (2009), no. 2, 591-643.

[35] A. Putman, The Johnson homomorphism and its kernel, preprint 2009.

[36] A. Putman, The Picard group of the moduli space of curves with level structures, to appear in Duke Math. J.

[37] A. Putman, The second rational homology group of the moduli space of curves with level structures, to appear in Adv. Math.

[38] A. Putman, Small generating sets for the Torelli group, to appear in Geom. Topol.

[39] M. Sato, The abelianization of the level $d$ mapping class group, J. Topol. 3 (2010), no. 4, 847-882.

[40] N. Wahl, Homological stability for mapping class groups of surfaces, to appear in the Handbook of Moduli.

Andrew Putman

Department of Mathematics

Rice University, MS 136

6100 Main St.

Houston, TX 77005

E-mail: andyp@rice.edu 